\title{
Research on Cooperative Innovation Behavior of Industrial Cluster Based on Subject Adaptability
}

\author{
Xiaohui Jia, Minghui Jiang, and Lei Shi \\ School of Management, Harbin Institute of Technology, Harbin 150001, China \\ Correspondence should be addressed to Minghui Jiang; mhjiang2000@sina.com
}

Received 31 March 2016; Revised 15 July 2016; Accepted 16 August 2016

Academic Editor: Alicia Cordero

Copyright (C) 2016 Xiaohui Jia et al. This is an open access article distributed under the Creative Commons Attribution License, which permits unrestricted use, distribution, and reproduction in any medium, provided the original work is properly cited.

\begin{abstract}
From the perspective of the interactive cooperation among subjects, this paper portrays the process of cooperative innovation in industrial cluster, in order to capture the correlated equilibrium relationship among them. Through the utilization of two key tools, evolutionary stable strategy and replicator dynamics equations, this paper considers the cost and gains of cooperative innovation and the amount of government support as well as other factors to build and analyze a classic evolutionary game model. On this basis, the subject's own adaptability is introduced, which is regarded as the system noise in the stochastic evolutionary game model so as to analyze the impact of adaptability on the game strategy selection. The results show that, in the first place, without considering subjects' adaptability, their cooperation in industrial clusters depends on the cost and gains of innovative cooperation, the amount of government support, and some conditions that can promote cooperation, namely, game steady state. In the second place after the introduction of subjects' adaptability, it will affect both game theory selection process and time, which means that the process becomes more complex, presents the nonlinear characteristics, and helps them to make faster decisions in their favor, but the final steady state remains unchanged.
\end{abstract}

\section{Introduction}

The root cause of interactive cooperation behaviors among subjects is through obtaining a higher profit to enhance their competitive advantage and ultimately ensure long-term survival and development. Innovation is an effective way to get profits, and the subjects should have a suitable model of innovation in order to pave the way for innovation. Cooperative innovation model has become quite common. However, the cluster itself has a very significant complex adaptive feature [1], so lacking the research on adaptability of subjects participated in cooperative innovation may lead to an unclear understanding of the decision-making game process of cooperative innovation.

1.1. Complex Adaptive System Theory and Adaptive Subject. When the innovation process of industrial cluster subjects is discussed, the methods presented are mostly following the equilibrium hypotheses and assuming that the economic players in the assumed equilibrium economic system have perfect rationality in behavior. With the applications of mathematics methodologies including regression analysis and mathematical analysis methods, analysis results are derived. The industrial cluster organism in the real world is definitely a nonlinear complex organism with numerous feedbacks, emergence, and other complex phenomena involved inherently. These problems mentioned above are obviously much more complicated and could not be explained well with traditional assumptions and analysis frames. However, Holland [2] from University of Michigan brought out the complex adaptive system (hereby CAS) theory which suggests that the features of adaptation might play a role in the mechanism of subject's development in the complex organism. The key point of the adaptive feature is that the subjects will communicate with environment and the other subjects in organism initiatively. During the process of communication, the subjects would learn from both environment and other subjects and accumulate learning experiences continuously. The subjects could change their internal structures and behavior manners according to the regulations learned and experiences accumulated from both the other subjects and the external environment. Through these interactions between each other, subjects in the sublevel of organism would prompt new structure, performance, and behavior 


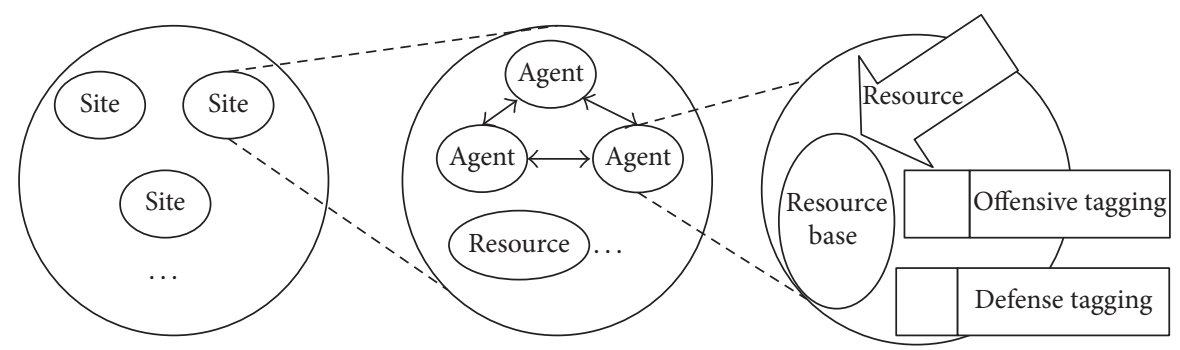

FIgURE 1: Schematic diagram of echo model. Source: $\mathrm{Hu}$ [6].

manners of the upper-level organism emergence, as well as the new performances that would be more complicated. The issues on industrial cluster innovation have been discussed in the study of Simon [3] who also investigated the subjects from the perspective of organism complexity and induced more researchers considering the issues $[4,5]$.

CAS theory considers that the individual initiative subjects would supply the evolution force to the entire industrial cluster organism. The self-driving subjects have the capacity of detecting both the circumstance and the other subjects around them initiatively. Based on these observations, they would make changes on their own behaviors to adapt to the environment and other subjects. These changes may lead to either success or failure, according to which the subjects would accumulate experiences. Thus, the core problem of CAS theory is to find out the characters and behavior patterns of these initiative adaptive subjects in complex organism. Meanwhile, the industrial cluster in reality can be considered as a huge complex organism consisting of individually initiative and adaptive subjects like the enterprises, government, colleges, and research institutes, as well as service mediations. From the functional view, the enterprise is the main innovative subject to carry on the function of manufacture innovation. The college and research institute are mainly responsible for scientific research and technology development, while their features of education function supply innovative human resource and human cultivation to the entire industrial cluster, so they are called the knowledge innovative subjects, while the main function of government is making relevant policy to inspire and encourage the other subjects to create diverse innovative activities. Thus the government is the institutional innovative subject. The intermediary agency plays the important part as the service innovative subject who provides the technology transfer and knowledge diffusion service. The enterprise plays the most important role in the industrial cluster activities, while the college, research institute, government, and intermediary agency are the supportive partners. Besides the enterprise, the other subjects also take part in the enterprise manufacture activities via production and research cooperation. The interactions between the subjects enforce the knowledge flows and technology diffusion inside the cluster. Furthermore, they enhance the effective allocation of innovative resources and make sure that the innovative capacity of the industrial cluster keeps increasing. From the microlevel, the subjects in the same cluster are posited in closer locations geographically and have the convenience in business connection and information communication. The convenience that is brought out from geographical location raises the significance of the competitive effect among enterprises, major subject, and other supportive subjects in the cluster, which finally forms the competition-cooperation mechanism to improve the successive possibility of innovative activities. Additionally, the competition-cooperation mechanism pushes the subjects to learn from innovative activities and transfer the competitive pressure into innovation force. Thereby, the improvement of adaptive capacity of the subject would enhance its survival from the fierce competitions. Obviously, learning is an important activity throughout the process of the competitive-cooperation mechanism. Thus, it also lightens the significance of the adaptive learning feature of the innovative subject.

With limited resources, cooperative innovation can increase productivity and create more significant innovation benefits. From the perspective of complex adaptive systems theory, cooperative innovation is conducive to enhance the adaptability of subjects in industrial clusters, which means that, after a period of adaptation, in order to overcome difficulties in independent innovation, the subjects take the initiative to find partners aiming at the creation and sharing of common value in terms of cooperative innovation. For specific searching process, the classic echo model in complex adaptive systems theory can help to understand two aspects. Firstly, when two bodies encounter in the same location, they will compare the offensive and defensive marks. Secondly, if the two have a perfect match, they will exchange resources, and the subject itself will make internal accumulation and integration of resources. If the resources reach certain level, the subject can breed new ones. Additionally, other ineffective storages and processing will lead to a small part in each other's database or even none [6], specifically shown in Figure 1.

1.2. Game Theory and Cooperative Innovation. Industrial cluster was formally put forward by Professor Michael Porter, in which the internal cooperation competition exists, especially cooperative competition mechanism among enterprises which has won the attention of numerous scholars both at home and abroad. In terms of cluster innovation, geographic concentration and the particularity of the network structure enable enterprises within the cluster to take the form of cooperative innovation so as to reduce the transaction cost [7], improve the efficiency of innovation [8], and have better access to external resources [9]. From microlevel, if each enterprise could make full use of cooperative innovation 
advantages in industrial cluster and be actively involved in the interaction, any of them can have better access to external knowledge, which is influenced by enterprise's technical learning ability [10], enterprise integration in research and development activities [11], the number of enterprises and partners in the alliance [12], mutual trust between each other [13], complementary level of their assets [14], participation and cooperation motivation factor [15], and so forth.

With the nature of game theory, using game theory to explore cooperative innovation of industrial cluster has become the most common method. In long-term repeated game, cooperation can bring higher benefits for enterprises, but in limited time game, to keep the stability of cooperation, punishing betrayal partners is a must. Enterprises choose betrayal because betrayal can bring more palpable benefits [16]. The Prisoner's Dilemma, Cournot Model, and other classic game models can be chosen according to different game situation in order to make a fitting depiction of cooperative innovation among the enterprises in industrial cluster. It can be found that if the enterprise betrays cooperation, the cooperative innovation will cease. To be more specific in the analysis of practical problems, you need to set up a more complex situation and introduce new conditions, with the aid of the experimental means such as repeated game so as to achieve the game equilibrium of enterprise innovation game within the cluster [17]. In addition to the transverse game mentioned above, the close link in the upstream and downstream industrial chain within an industrial cluster has promoted some scholars to study competition and cooperation among enterprises in the upstream and downstream industrial chain [18].

However, studies have shown that cooperation form of the subjects in type of Prisoner's Dilemma cannot be maintained long in reality. Therefore, to keep stable cooperation in a classic Prisoner's Dilemma game, we cannot simply consider economic factors, and more other factors should be added [19]. Therefore, this paper will introduce the adaptability of game subjects based on classical evolutionary game model and build a stochastic dynamic game process to analyze the complex adaptability shown in the process of the game in industrial cluster innovation. The contents are arranged as follows. Section 2 is the introduction of evolutionary game theory. Section 3 is the construction and application of the evolutionary game theory model for cooperative innovation in industrial clusters. Section 4 is the construction and application of the stochastic evolutionary game theory model for cooperative innovation in industrial clusters. Section 5 is a case study and Section 6 is the conclusion.

\section{Introduction of Evolutionary Game Theory}

Classical game theory suggests that individuals have superrational characteristics and have all the information of the payoff matrix. Therefore, under such conditions, subjects who participate in the game will finally choose Nash equilibrium strategy. But in reality, the limit of the individual's ability cannot guarantee that the best strategy is adopted in complex environments and neither can maximum benefits be guaranteed. In this case, the individual will generally choose their own "satisfied" game strategy through heuristic method based on the partial information that they get. At this time, the individual's choice has limited rationality (bounded rationality), which can be analyzed using evolutionary game theory method.

When it comes to the cooperative innovation behaviors among subjects in industrial clusters, the use of evolutionary game theory is built on the heterogeneity of the subjects and the nonlinear interaction among them, which make such cooperative innovation exhibit the characteristics of complex open systems. It means that the subject generates interactions and creates effects of innovative cooperation with others, which based on the innovation factors, and this effect cannot be produced by a single body. To be more specific, in the internal innovation system of industrial clusters, specialization and complementary resources lay the foundation for cooperative innovation among the subjects; technological innovation complexity sets a higher requirement for capital, facilities, and others in innovation activities; therefore innovation itself becomes a complicated systematic project beyond a single body. The above characteristics are particularly significant in the innovation process of industrial clusters, in which enterprises, universities, and research institutions conduct cooperative innovation in the common pursuit laying the foundation for the formation and development of innovation networks, as well as ensuring that industrial cluster innovation system will self-mutate and bring a new structure emerging from disorder to order. For subjects in industrial cluster, a large number of heterogeneous bodies possess the characteristics of bounded rationality, which means that innovation strategy selection is also the learning process. With the above analysis, cooperative innovation behaviors in industrial clusters among subjects can be studied by using evolutionary game analysis framework, in which evolutionary stable strategy and replicator dynamic equations are two important concepts.

2.1. Evolutionary Stable Strategy. Evolutionary stable strategy, ESS for short, was first presented by Smith and Price [20] in the study of evolutionary biology. Its basic idea is that the equilibrium can resist invasion by variations, with the hypothesis of symmetric game by people picked from a large overall repeated random selection in the process of evolutionary game. It is assumed that all of the participants are required to adopt a certain strategy from the start of the game. Now some of the individuals are to take another strategy that is predetermined. If the population strategy can resist any invasion of small variations, it means that the original population selection strategy will bring higher payoff than that of the variations; on occasion, under the natural pressure, variations can either change strategy to adapt to the original population or quit the game to be eliminated in the process of evolution. To enable the evolutionary game theory for the sake of obtaining more general application, Weibull [21] abstracted it into mathematical expression. A more detailed description is as follows.

Supposing that a pure strategy set is expressed as $K=$ $\{1,2, \ldots, k\}$, then the matching mixed strategy set can be 
expressed as $\Delta=\left\{x \in R_{+}^{k}: \sum_{i \in K} x_{i}=1\right\}$, and its polyhedron can be expressed as $\Theta=\Delta^{2}$. When one party in the game takes $y(y \in \Delta)$ as the strategy, the other takes $x(x \in \Delta)$ as its strategy, and the payoff can be expressed as $u(x, y)=$ $x \cdot A y$, in which $A$ represents payoff matrix of the game player. According to the other's strategy $y, y \in \Delta$, one's optimal strategy is $x \in \Delta$, which can be expressed as $\beta^{*}(y) \subset \Delta$.

Assume that some members in the group take the same initial strategy (pure strategy and mixed strategy), $x \in \Delta$, while variations in the group take mutation strategy (pure strategy and mixed strategy), $y \in \Delta$, in which the proportion of variations in the group can be expressed as $\varepsilon(\varepsilon \in(0,1))$. Extract individuals from the binary group randomly and repeatedly to pair game in which the probability of each individual is pumped are equivalent. Therefore, through extracting each individual in the group for game, the probability of the chosen one that takes the mutation strategy $y$ can be expressed as $\varepsilon$, and its probability of taking the original strategy can be expressed as $1-\varepsilon$. To demonstrate the mixed strategy status, we can come to

$$
\omega=\varepsilon y+(1-\varepsilon) x, \quad(\omega \in \Delta) .
$$

The average payoff of existing strategy in the group game is expressed as $u(x, w)$ and that of mutation strategy is expressed as $u(y, w)$, when the average payoff of choosing mutation strategy is lower than that of the existing strategy; namely,

$$
u[x, \varepsilon y+(1-\varepsilon) x)>u[y, \varepsilon y+(1-\varepsilon) x] .
$$

If this kind of inequality $(y \neq x)$ is maintained and $\varepsilon$ is small enough, then the strategy $x \in \Delta$ is called evolutionary stable. If for any $y \neq x, \forall \bar{\varepsilon}_{y} \in(0,1)$, formula (2) exists when the condition $\varepsilon \in\left(0, \bar{\varepsilon}_{y}\right)$ can be satisfied, then $x \in \Delta$ is an evolutionary stable strategy, namely, the ESS.

All evolutionary stable strategies can be listed as $\Delta^{\text {ESS }} \subset \Delta$; of course, this collection could also be empty. Any strategy contained within the collection is the best choice for the individual; if strategy $x$ falls into nonoptimal situation, there must be another strategy $y$ which means that the individual can obtain higher returns. If the mutation strategy occupies a small amount in the entire population, according to the continuity of $u$, strategy $y$ yields have higher returns than strategy $x$. In this case, $x$ does not have evolutionary stability, so we can get

$$
\Delta^{\mathrm{ESS}} \subset \Delta^{\mathrm{NE}} \text {. }
$$

In formula (3), $\Delta^{\mathrm{NE}}$ represents the symmetric Nash equilibrium.

Meanwhile, compared with Nash equilibrium, evolutionary stable strategy has more strict limits, which means that if strategy $x$ is evolutionarily stable, then it will be more competitive than the mutation strategy $y$, and it can be expressed as

$$
\Delta^{\mathrm{ESS}}=\left\{x \in \Delta_{\mathrm{NE}}: u(y, y)<u(x, y)\right\} .
$$

According to what has been discussed, the evolutionary stable strategy can be expressed in other ways, only if

$$
\begin{aligned}
& u(y, x) \leq u(x, x) \quad \forall y \\
& u(y, x)=u(x, x) \Longrightarrow \\
& u(y, y)<u(x, y) \quad \forall y \neq x .
\end{aligned}
$$

Therefore, the deduction is that strategy $x$ is evolutionary stable, which is in accordance with what Smith and Price originally defined.

2.2. Replicated Dynamic Equation. In the repeated dynamic game, assuming that two types of participants both take pure strategy, the pure strategy set is named $S . \varphi_{t}(s)$ represents the set of participants who take pure strategy $s \in S$ at stage $t . \theta_{t}(s)$ represents the vector of proportion of those participants who take pure strategy $s$; then formula can be reckoned as

$$
\theta_{t}(s)=\frac{\varphi_{t}(s)}{\sum_{r \in S} \varphi_{t}(r)} .
$$

The expected utility of the participants who take pure strategy $s$ at stage $t$ is

$$
u_{t}(s)=\sum_{r \in S} \theta_{t}(r) \cdot u(s, r),
$$

in which $u(s, r)$ represents the expected utility of the participants who take pure strategy $s$ when participants of the other type take pure strategy $r$. Then the average expected utility of the group is

$$
\bar{u}_{t}=\sum_{s \in S} \theta_{t}(s) \cdot u(s) .
$$

According to continuous-time replicator dynamic model put forward by Taylor and Jonker [22], it can calculated as

$$
\varphi_{t}^{\prime}(\mathrm{s})=\varphi_{t}(s) \cdot u_{t}(s)
$$

After derivation of formula (6), the equation can be inferred as

$$
\frac{d \theta_{t}(s)}{d t}=\frac{\varphi_{t}^{\prime}(s) \sum_{r \in S} \varphi_{t}(r)-\varphi_{t}(s) \sum_{r \in S} \varphi_{t}^{\prime}(r)}{\left|\sum_{r \in S} \varphi_{t}(r)\right|^{2}} .
$$

Through substitution of (9), (10) can be simplified as

$$
\frac{d \theta_{t}(\mathrm{~s})}{d t}=\theta_{t}(s)\left|u_{t}(s)-\bar{u}_{t}\right| .
$$

Formula (11) is the replicated dynamic equation. Assume that it is zero, which is $d \theta_{t}(\mathrm{~s}) / d t=0$. Then all the stable states can be calculated. According to the stability theorem of differential equation, the stability of its steady state neighborhood can be discussed, which is the stable state of stability when tiny deviation disturbance occurs. A steady state $\theta_{t}^{\prime}(\mathrm{s})$ must have robustness to small perturbations, and then it can be regarded as evolutionary stable strategy. 


\section{Evolutionary Game Model of Cooperative Innovation in Industrial Cluster}

Through a thorough analysis of the basic concepts in industrial clusters, enterprises are the main forces in the innovation activities within the cluster [23]. Thus, to simplify the model, this paper only focuses on the evolutionary game process of cooperative innovation among subjects in quantitative calculation. In real industrial cluster, enterprises who are in the completely same size are rare. Their differences in size can be commonly seen. This paper divides enterprises into two categories, which named large ones and SMEs, and sets the model parameters considering their unequal position in innovation.

\subsection{Construction of Evolutionary Game Model of Cooperative} Innovation. Enterprises in industrial cluster have the nature of the nonzero-sum game and repeated game. Suppose that there exist a large enterprise group 1 and a SME group 2, which are regarded as the players of cooperative innovation. According to the evolutionary game theory, enterprises from group 1 and group 2 are randomly selected using the pairing mode. The two enterprises selected are referred to as enterprise 1 and enterprise 2 . Therefore, this paper builds the game model considering the following factors.

(1) Participants in the Game. Assume that enterprise 1 is a large enterprise which intends to innovate within a cluster and enterprise 2 is a SME which intends to imitate others' pathway. The ratio of assets of enterprise 1 in the cluster is $\beta$, and that of enterprise 2 is $1-\beta$. Large enterprises are wellfunded, having large scale and advantages in brand effect, technical skills, management experience, and so on, while SMEs usually have small scale and lacking funds and are less competitive in brand, technology, management, and so forth.

(2) Behaviors. If no enterprises take cooperative innovation, the normal payoffs of them are $r_{1}$ and $r_{2}$. If they take cooperative innovation, their payoff is $B$, in which the payoff is distributed according to their proportion in the cluster, and large enterprises get $\alpha B$, while SMEs get $(1-\alpha) B$. The innovation input costs are $c_{1}$ and $c_{2}$, respectively. The amount of support from the government is $S$. In accordance with the proportion of their assets, they each get $\beta S$ and $(1-\beta) S$.

(3) Strategy. If enterprises in the cluster have mutual trust and take cooperation behavior before $n$ times' cooperative innovation, each enterprise continues to take the cooperation strategy. But once an enterprise within the cluster betrays in stage $n$, other enterprises in the cluster will no longer cooperate in the future.

According to the above assumptions of the game elements, payoff matrix of two enterprises' cooperative innovation can be constructed as shown in Table 1 .

Through further analysis of behaviors of enterprises 1 and 2 and payoffs in the table, the equations can be obtained:

Case 1: $E_{11}=r_{1}-c_{1}+\alpha B+\beta S$,

$$
E_{12}=r_{2}-c_{2}+(1-\alpha) B+(1-\beta) S
$$

TABLE 1: The payoff matrix of cooperative innovation.

\begin{tabular}{lccc}
\hline & \multirow{2}{*}{ State (probability) } & \multicolumn{2}{c}{ Enterprise 2 } \\
& & Collaboration & Betrayal \\
\hline \multirow{2}{*}{ Enterprise 1} & Collaboration & $E_{11}, E_{12}$ & $E_{21}, E_{22}$ \\
& Betrayal & $E_{31}, E_{32}$ & $E_{41}, E_{42}$ \\
\hline
\end{tabular}

Case 2: $E_{21}=r_{1}-c_{1}$,

$$
E_{22}=r_{2}
$$

Case 3: $E_{31}=r_{1}$,

$$
E_{32}=r_{2}-c_{2}
$$

Case 4: $E_{41}=r_{1}$,

$$
E_{42}=r_{2} \text {. }
$$

3.2. Analysis of Evolutionary Game Model of Cooperative Innovation. According to the core of evolutionary game theory, the analysis is mainly from the replicated dynamic equations and evolutionarily stable strategies.

3.2.1. Replicated Dynamic Equation. Assuming that the proportion of large enterprises selecting cooperative innovation strategy is $x$, then the proportion of selecting noncooperative innovation strategy is $1-x$. The proportion of SMEs selecting construction strategy is $y$, and then the proportion of selecting nonconstruction strategy is $1-y$.

The payoff of large enterprises selecting cooperative innovation strategy is

$$
U_{1}=y\left(r_{1}-c_{1}+\alpha B+\beta S\right)+(1-y)\left(r_{1}-c_{1}\right) .
$$

The payoff of large enterprises selecting noncooperative innovation strategy is

$$
U_{1}^{\prime}=y r_{1}+(1-y) r_{1}
$$

The average payoff of large enterprises selecting cooperative innovation strategy is

$$
\begin{aligned}
U= & x U_{1}+(1-x) U_{1}^{\prime} \\
= & x \cdot\left[y\left(r_{1}-c_{1}+\alpha B+\beta S\right)+(1-y)\left(r_{1}-c_{1}\right)\right] \\
& +(1-x) \cdot\left[y r_{1}+(1-y) r_{1}\right] \\
= & x y(\alpha B+\beta S)-x c_{1}+r_{1} .
\end{aligned}
$$

Meanwhile, it can be deducted as

$$
\begin{aligned}
U_{1}-U= & y\left(r_{1}-c_{1}+\alpha B+\beta S\right)+(1-y)\left(r_{1}-c_{1}\right) \\
& -\left[x y(\alpha B+\beta S)-x c_{1}+r_{1}\right] \\
= & (1-x)\left[y(\alpha B+\beta S)-c_{1}\right] .
\end{aligned}
$$

The same is true for the payoff of SMEs:

$$
\begin{aligned}
U_{2}= & x\left(r_{2}-c_{2}+(1-\alpha) B+(1-\beta) S\right) \\
& +(1-x)\left(r_{2}-c_{2}\right)
\end{aligned}
$$




$$
\begin{aligned}
U_{2}^{\prime} & =x r_{2}+(1-x) r_{2} \\
U & =y U_{2}+(1-y) U_{2}^{\prime} \\
U_{2}-U & =(1-y)\left\{x[(1-\alpha) B+(1-\beta) S]-c_{2}\right\} .
\end{aligned}
$$

For any party in the game, their probability of taking a certain strategy changes with the time, and the dynamic changing process of probability depends on the speed of its dynamic change. According to the basic principle of replicated dynamic equation, the dynamic changing speed is associated with the probability of a certain strategy taken by the enterprise last time and difference between the benefits and the average revenue under this strategy. By the definition of the replicated dynamic equation, the replicated dynamic equations for large enterprises and SMEs choosing construction strategy are

$$
\begin{aligned}
F(x) & =\frac{d x}{d t}=x\left(U_{1}-U\right) \\
& =x(1-x)\left[y(\alpha B+\beta S)-c_{1}\right]
\end{aligned}
$$

$$
\begin{aligned}
F(y) & =\frac{d y}{d t}=y\left(U_{2}-U\right) \\
& =y(1-y)\left\{x[(1-\alpha) B+(1-\beta) S]-c_{2}\right\} .
\end{aligned}
$$

The above formulas show the dynamic changing speed of large enterprises and SMEs adopting construction strategies, which together constitute the whole dynamic of the system. Therefore, only if $x=0,1$ or $y=y^{*}=c_{1} /(\alpha B+\beta S)$, the proportion of large enterprises which takes construction strategy is stable. There is no exception with that of SMEs; only if $y=0,1$ or $x=x^{*}=c_{2} /((1-\alpha) B+(1-\beta) S)$, the proportion of SMEs which takes construction strategy is stable.

3.3. Evolutionary Stable Strategy. According to the method proposed by Friedman [24], the stability of the dynamic equilibrium of the group described by the differential equation can be judged by local stability of Jacobian matrix. The Jacobian matrix is as follows:

$$
J=\left\{\begin{array}{ll}
a & b \\
c & d
\end{array}\right\}=\left\{\begin{array}{cc}
(1-2 x)\left[y(\alpha B+\beta S)-c_{1}\right] & x(1-x)\left[(\alpha B+\beta S)-c_{1}\right] \\
y(1-y)\left\{[(1-\alpha) B+(1-\beta) S]-c_{2}\right\} & (1-2 y)\left\{x[(1-\alpha) B+(1-\beta) S]-c_{2}\right\}
\end{array}\right\} .
$$

In order to study the stability of the equilibrium of differential equation, the following variables can be defined. By the matrix trace, the formula can be got as follows:

$$
\operatorname{tr} J=a+d \text {, defining } p=-\operatorname{tr} J=-(a+d) .
$$

Determinant of the matrix is $q=\operatorname{det} J=a * d-c * b$.

According to the stability theory of the equilibrium point of differential equation, the theorem is as follows:

$$
\begin{aligned}
& \text { If } p>0 \text { and } q>0 \text {, then the equilibrium point } P_{0}\left(x_{0}\right. \text {, } \\
& \left.y_{0}\right) \text { is stable. } \\
& \text { If } p<0 \text { or } q<0 \text {, then the equilibrium point } P_{0}\left(x_{0}, y_{0}\right) \\
& \text { is unstable. }
\end{aligned}
$$

To be more specific, the existence of the equilibrium point and its stability mainly refer to the costs and benefits in the process of innovation. From different costs and benefits of both parties of the game, the following part will further analyze the equilibrium and its stability under different conditions.

Situation 1. If $\alpha A+\beta B<c_{1}$ and $(1-\alpha) A+(1-\beta) B<c_{2}$, it means that innovation revenue of both parties in the game is less than their costs of inputs, and the formula can be deducted as $x=c_{2} /((1-\alpha) A+(1-\beta) B)>1$ and $y=c_{1} /(\alpha A+\beta B)>1$. Under such circumstance, there are four equilibrium points, which are $(0,0),(0,1),(1,0)$, and $(1,1)$. According to the judgment method of the stability of differentiation equation, the results are as follows in Table 2.
From Table 2 , saddle points $(0,1)$ and $(1,0)$ exist under such circumstance, unstable point $(1,1)$ and stable point $(0,0)$, which means that $(0,0)$ is the evolutionary stable strategy of both large enterprises and SMEs in the game. In this case, both parties of the game will eventually choose noncooperative innovation strategy. Replication dynamics and its stability diagram of both parties can be drawn in the game, as shown in Figure 2.

Following the same steps, the rest three situations can be analyzed. Accordingly, their stable results can be shown from Table 3.

The phase diagrams are as shown in Figure 3.

From Figure 3, Situation 2 and Situation 3 will eventually converge to $(0,0)$, but Situation 4 is more complex. In this specific analysis, according to the direction of the arrows in the above diagram, the game of cluster innovation, $\left[x^{*}=0, y^{*}=0\right]$ and $\left[x^{*}=1, y^{*}=1\right]$ are the ESS evolutionary stable strategies of this game. When the initial situation falls in $E_{1} E_{2} P E_{4}$ area, it will eventually converge to evolutionary stable strategy $\left[x^{*}=0, y^{*}=0\right]$, namely, taking noncooperative innovation strategy. When initial situation falls in $E_{2} P E_{4} E_{3}$ area, it will eventually converge to evolutionary stable strategy $\left[x^{*}=1, y^{*}=1\right]$, namely, taking cooperative innovation strategy. For further discussing cooperative innovation, $\alpha A+\beta B>c_{1}$ and (1$\alpha) A+(1-\beta) B>c_{2}$ must be met. The basic requirement of cooperative innovation strategies taken by both parties in the game is that innovation benefits must be greater than the cost. 
TABLE 2: Results of the stability judgment of Situation 1.

\begin{tabular}{lccc}
\hline Equilibrium point & $Q$ & $P$ & Stability \\
\hline$(0,0)$ & Above 0 & Above 0 & Local stability point \\
$(0,1)$ & Below 0 & Uncertain & Saddle point \\
$(1,0)$ & Below 0 & Uncertain & Saddle point \\
$(1,1)$ & Above 0 & Above 0 & Unstable \\
\hline
\end{tabular}

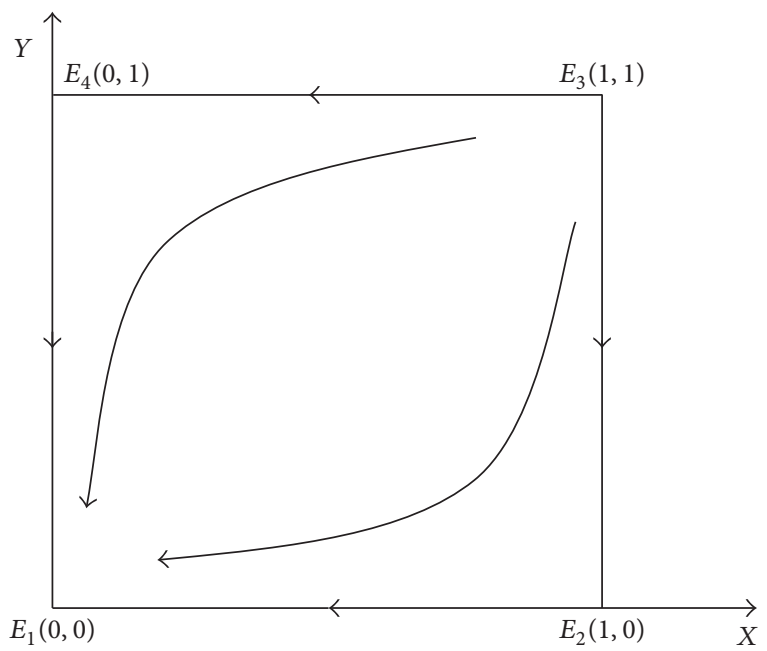

Figure 2: Situation 1: $\alpha A+\beta B<c_{1},(1-\alpha) A+(1-\beta) B<c_{2}$.
TABLE 3: The results of the other three situations.

\begin{tabular}{lc}
\hline Condition & Stable point \\
\hline Situation 2: $\alpha B+\beta S<c_{1},(1-\alpha) B+(1-\beta) S>c_{2}$ & $(0,0)$ \\
Situation 3: $\alpha B+\beta S>c_{1},(1-\alpha) B+(1-\beta) S<c_{2}$ & $(0,0)$ \\
Situation 4: $\alpha B+\beta S>c_{1},(1-\alpha) B+(1-\beta) S>c_{2}$ & $(0,0)$ and $(1,1)$
\end{tabular}

Meanwhile, to discuss the probability of taking cooperative innovation strategy in Situation 4, from Figure 3, we know that the greater the area of the $E_{2} P E_{4} E_{3}$, the greater the probability both sides of the game may choose $(1,1)$, which is, namely, more likely to take cooperative innovation strategy. The position of $P\left(x^{*}, y^{*}\right)$ determines the size of this part. According to $y=c_{1} /(\alpha B+\beta S), x=c_{2} /((1-\alpha) B+$ $(1-\beta) S)$, we can get $y=1-\left(\alpha B+\beta S-c_{1}\right) /(\alpha B+\beta S)$, $x=1-\left([(1-\alpha) B+(1-\beta) S]-c_{2}\right) /[(1-\alpha) B+(1-\beta) S]$, and we know that the greater $\alpha B+\beta S-c_{1},[(1-\alpha) B+(1-$ $\beta) S]-c_{2}$, the greater the area of $E_{2} P E_{4} E_{3}$, which means the higher probability of taking cooperative innovation. Thus, the cooperation between innovation subjects depends on excess profits and their initial state.

Lastly, we will discuss the equilibrium point $P\left(x^{*}, y^{*}\right)$. According to $y^{*}=c_{1} /(\alpha B+\beta S)$ and $x^{*}=c_{2} /((1-\alpha) B+$ $(1-\beta) S)$, the Jacobian matrix is as follows:

$$
J=\left\{\begin{array}{ll}
a & b \\
c & d
\end{array}\right\}=\left\{\begin{array}{cc}
0 & x(1-x)\left[(\alpha B+\beta S)-c_{1}\right] \\
y(1-y)\left\{[(1-\alpha) B+(1-\beta) S]-c_{2}\right\} & 0
\end{array}\right\} .
$$

The matrix eigenvalues are $\lambda_{1}=\sqrt{A B}$ and $\lambda_{2}=-\sqrt{A B}$, in which $A=x(1-x)\left[(\alpha B+\beta S)-c_{1}\right], B=y(1-y)\{[(1-$ $\left.\alpha) B+(1-\beta) S]-c_{2}\right\}, 0<x, y<1, \alpha B+\beta S-c_{1}>0$, $[(1-\alpha) B+(1-\beta) S]-c_{2}>0$. Therefore, $A>0, B>0$, and $A B>0$, which mean $\lambda_{1}$ and $\lambda_{2}$ are positive and negative roots, respectively, and $P\left(x^{*}, y^{*}\right)$ is just a saddle point instead of a locally asymptotically stable point.

3.4. Application of Evolutionary Game Model of Cooperative Innovation. By building a model of evolutionary stable strategies and making a mathematical analysis, some parameters are set and specific research is carried out using computer simulation method to depict dynamic evolution. Based on the game system in formula (18), Matlab 2014a is used in this paper for the simulation of the game process.

Firstly, we assume that the cost of cooperative innovation is $c_{1}=10, c_{2}=2.5$. The payoff of cooperative innovation is $B=4$. The distribution proportion for large enterprise is $\alpha=0.7$. Support from the government is $S=3$, and the distribution proportion for large enterprise is $\beta=0.6$. We set the initial value as $x=0.6, y=0.7$; then the differentiation equation above can be transformed into

$$
\begin{aligned}
& F(x)=\frac{d x}{d t}=x(1-x)(4.6 y-10) \\
& F(y)=\frac{d y}{d t}=y(1-y)(2.4 x-2.5)
\end{aligned}
$$

The result is shown in Figure 4, from which we can see that both sides of the game take noncooperative innovation strategy.

From Figure 4, the final equilibrium point of the game is $(0,0)$, which means that both sides refuse to take noncooperative innovation. And $\alpha A+\beta B=0.7 \times 4+0.6 \times 3=4.6<c_{1}=10$, $(1-\alpha) A+(1-\beta) B=0.3 \times 4+0.4 \times 3=1.2+1.2=2.4<c_{2}=2.5$, which coincides with the analysis in Section 3.2 that there exists one equilibrium point $(0,0)$.

Secondly, we reduce the cost of cooperative innovation to $c_{1}=2, c_{2}=0.5$; ceteris paribus, then the differentiation equation in step 1 can be changed into

$$
\begin{aligned}
& F(x)=\frac{d x}{d t}=x(1-x)(4.6 y-2) \\
& F(y)=\frac{d y}{d t}=y(1-y)(2.4 x-0.5) .
\end{aligned}
$$

Then we get the result as Figure 5 .

From Figure 5, after reducing the cost, both sides take cooperative innovation strategy. $\alpha A+\beta B=0.7 \times 4+0.6 \times 3=$ $4.6>c_{1}=2,(1-\alpha) A+(1-\beta) B=0.3 \times 4+0.4 \times 3=1.2+1.2=$ $2.4>c_{2}=0.5$. From Section 3.2, there exist two equilibrium points $(0,0)$ and $(0,1)$, which is in accordance with previous conclusions.

Thirdly, based on step 2, we increase the distribution proportion of cooperative innovation to $B=8$ and raise 


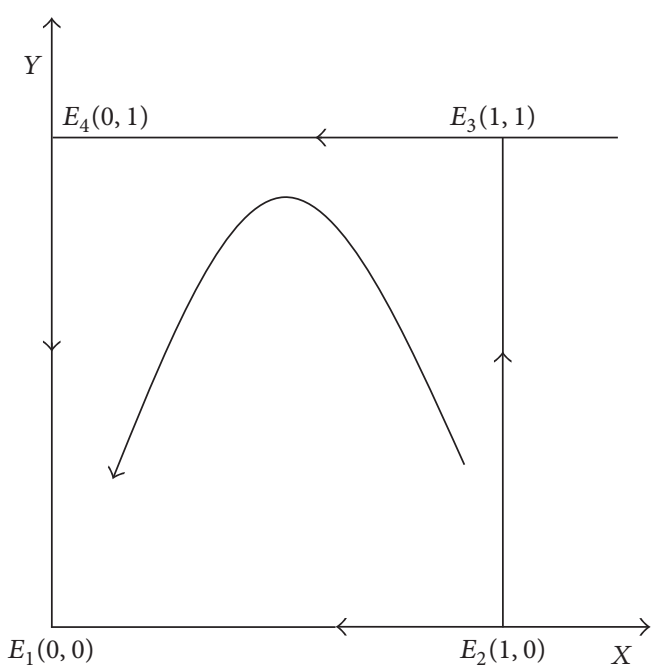

(a) Situation 2: $\alpha B+\beta S<c_{1},(1-\alpha) B+(1-\beta) S>c_{2}$

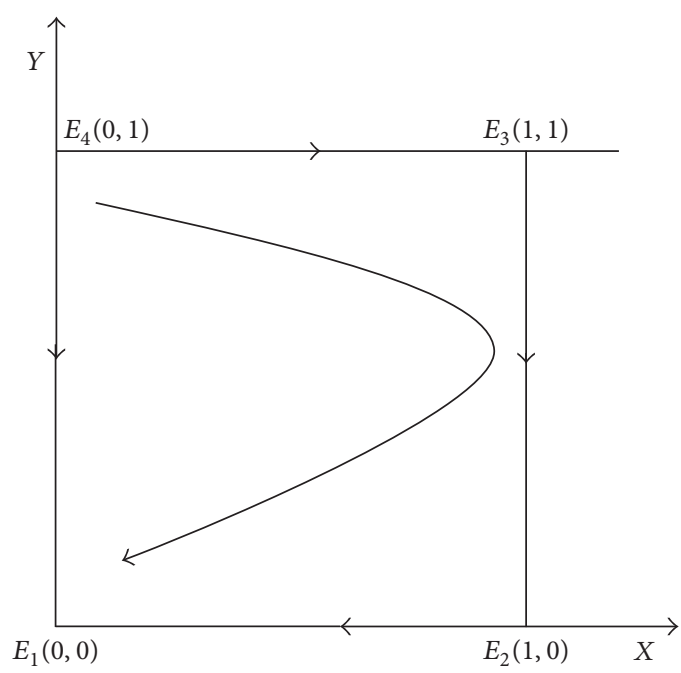

(b) Situation 3: $\alpha B+\beta S>c_{1},(1-\alpha) B+(1-\beta) S<c_{2}$

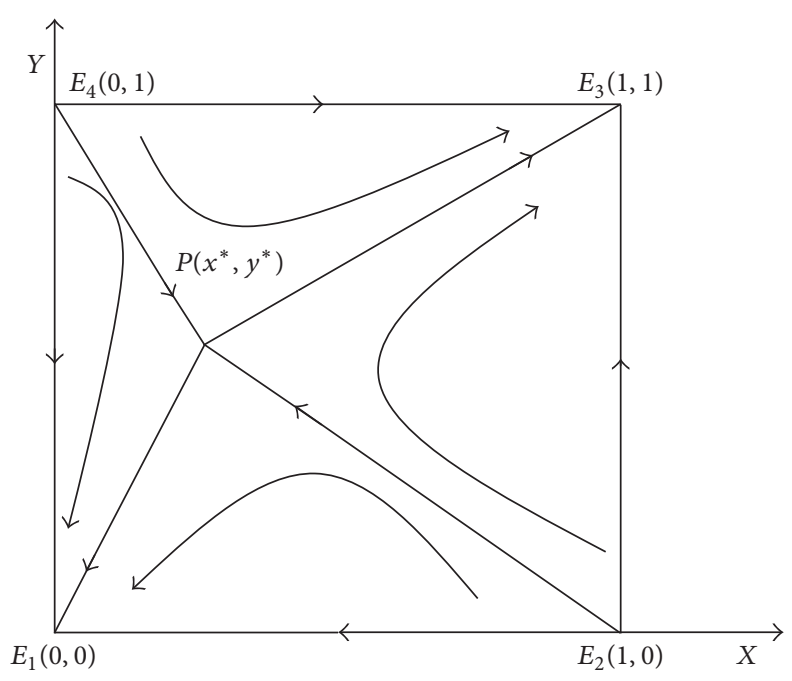

(c) Situation 4: $\alpha B+\beta S>c_{1},(1-\alpha) B+(1-\beta) S>c_{2}$

FIgURE 3: The phase diagrams of the other three situations.

the distribution proportion of government support to $S=6$. Ceteris paribus, then the differentiation equation in step 2 can be changed into

$$
\begin{aligned}
& F(x)=\frac{d x}{d t}=x(1-x)(9.2 y-2) \\
& F(y)=\frac{d y}{d t}=y(1-y)(4.8 x-0.5)
\end{aligned}
$$

The figure is shown in Figure 6.

At this time, $\alpha A+\beta B=0.7 \times 8+0.6 \times 6=9.2>$ $c_{1}=2,(1-\alpha) A+(1-\beta) B=0.3 \times 8+0.4 \times 6=$ $4.8>c_{2}=0.5$. From Figure 6 , in this case both sides do not only choose the cooperative innovation strategy, and their time to achieve the equilibrium is shorter than that in the second step. It shows that both sides of the game can choose cooperative innovation strategy more quickly under more favorable innovation conditions.
After the analysis of results of Figures 4, 5, and 6 we can see that the cost and benefits of cooperative innovation and the amount of government support will have an impact on the final game stable state of the interactive cooperation among the subjects. With lower cost, greater benefits, and more amount of government support, the subjects will be more likely to choose cooperative innovation strategy. Meanwhile, this paper selects cooperative innovation costs, benefits, and government support as three factors in the game, which has a certain universality. Thus the model results can be applied to more types of industrial clusters subjects, namely, for any participant in the cooperative innovation activities selecting cooperation strategy; if the innovation cost is more than the benefit from innovation, the whole cooperative innovation will gradually evolve to select "noncooperative innovation." This will have an adverse impact on coevolution development among subjects in industrial cluster. 


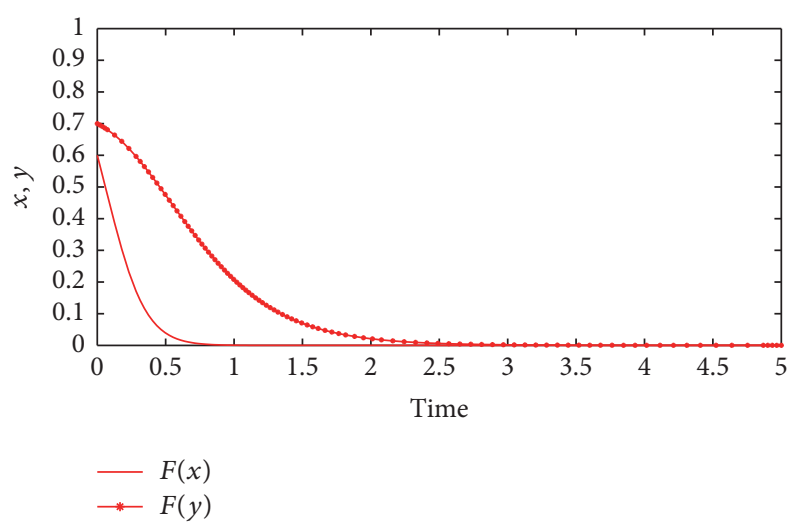

FIgURE 4: The equilibrium figure after 1st step game.

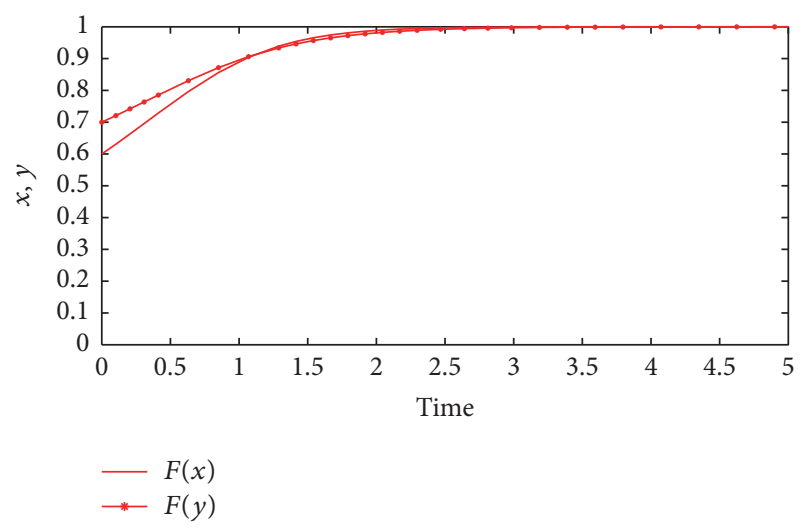

FIGURE 5: The equilibrium figure after 2nd step game.

\section{Stochastic Evolutionary Game Model of Cooperative Innovation of Industrial Cluster}

Based on the evolution game model in Section 3, according to what has been set previously, participants in the game are large enterprises and SMEs, which are not identical in knowledge structure, learning ability, and other aspects of internal properties. Therefore, the game system in Section 3 is just from the perspective of the cost-benefit of innovation and focuses more on some deterministic conditions. If the intrinsic properties of the enterprises are involved into the game system, then further analysis has to be made considering the influence of the differences on the game process and the final game strategy selection.

4.1. Subject Adaptability and Stochastic Differential Equation. The existing study on evolutionary game of cooperative innovation is tantamount to explore the dynamic equilibrium from external conditions such as input-output analysis. But the subjects in industrial clusters are adaptive bodies, and their own properties have an important influence on the evolutionary game process. So this section will combine subjects' adaptability and stochastic differential equations to

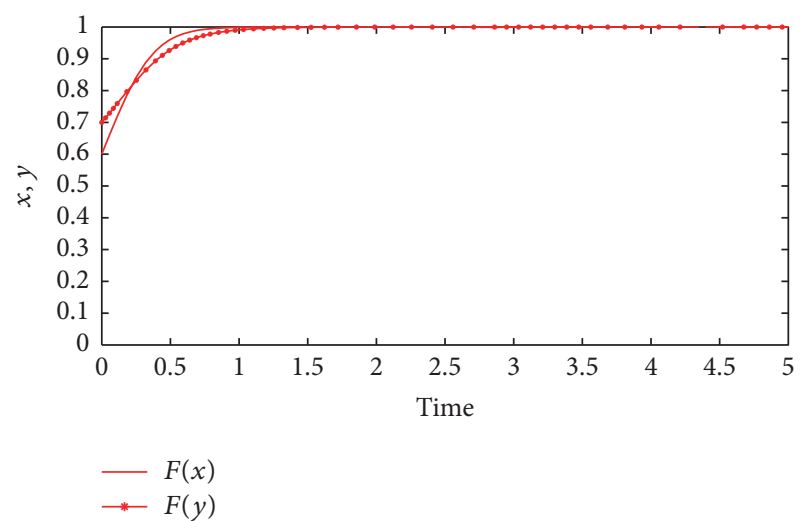

FIGURE 6: The equilibrium figure after 3rd step game.

construct a more realistic model of stochastic evolutionary game model.

In terms of the association between adaptability and evolutionary dynamics, Ewens [25] can be quoted to best fit; Wright proposed a three-phase process under which evolution could most easily occur. This view assumes that a large population is normally split up into three semiisolated subpopulations, each of which is comparatively small in size. Within each, there exists a genotypic fitness surface, depending on the genetic constitution of many loci. Besides, in conformity with the "increase in fitness value" concept, gene frequencies tend to move so that regional peaks in this surface can be approached. The surface of mean fitness is assumed to be very complex with a multiplicity of local maxima, and some are even higher than others. If it is only a full deterministic behavior, then the system simply moves to the nearest selective peak and remains there. The comparatively small size can guarantee that such strict deterministic behavior does not occur: random drift can move gene frequencies across a saddle and possibly under the control of a higher selective peak, which laid the foundation for the research on the relationship between biological evolution and the potential function. Meanwhile, foreign scholars such as Ao [26,27] also studied evolutionary dynamics from the ecological point of view to build a bridge between adaptability and stochastic differential equations. In addition, Song [28] and Ding [29] from Shanghai Jiaotong University also made a further study into it, so specific mathematical derivation is omitted here.

Based on the fruitful results in evolutionary dynamics, this paper will connect dynamic evolutionary processes of adaptive subjects and stochastic differential equations, which pave the way for the introduction of subjects' adaptability into stochastic evolutionary game system.

4.2. Analysis of Stochastic Evolutionary Game Model of Cooperative Innovation. First, in order to facilitate the impact analysis of introduction of adaptability, we deform formula (18) and build a deterministic system,

$$
\begin{aligned}
& d x(t)=x(1-x)(a y+b) d t \\
& d y(t)=y(1-y)(c x+d) d t .
\end{aligned}
$$


We just select one equation from the system,

$$
d x(t)=x(1-x)(a y+b) d t .
$$

Through analysis, the analytical method is the same as that of $d y(t)=y(1-y)(c x+d) d t$, so we omit the process.

Its potential function is as follows:

$$
\begin{aligned}
U(x) & =-\int_{0}^{x} t(1-t)(a y+b) d t \\
& =\frac{a y+b}{6}\left(3 x^{2}-2 x^{3}\right) .
\end{aligned}
$$

According to what has been discussed, the extreme value of $U(x)$ depends on the value of $a y+b$ in the place, which means that qualitative change of $U(x)$ occurs when $a y+b=0$. If $a y+b>0, U(x)$ takes minimum when $x=1$ and maximum when $x=0$. If $a y+b<0, U(x)$ takes minimum when $x=$ 0 and maximum when $x=1$. Therefore, when the value of $a y+b$ changes from positive to negative, the transition of the deterministic system changes from $x=1$ to $x=0$.

Next, we will introduce the adaptability in biology into the deterministic system and build the following stochastic dynamic system:

$$
\begin{aligned}
d x(t)= & x(1-x)(a y+b) d t \\
& +x(1-x) y d w_{1}(t) \\
d y(t)= & y(1-y)(c x+d) d t \\
& +y(1-y) y d w_{2}(t)
\end{aligned}
$$

$d w_{1}(t) d w_{2}(t)=d t$

Among them $a, b, c$, and $d$ are all constants, and $x, y \in$ $[0,1]$. Here, this paper focuses on the economic meaning behind $w_{1}(t)$ and $w_{2}(t)$ : during the cooperative innovation among subjects in industrial clusters, many restrictions jointly play a very important role, including not only the ability, quality, and other intrinsic properties, but also social environment and even culture and other noneconomic factors. Although the salient features of these factors cannot be accurately measured, certain statistical distribution exists, which is one of the main behavioral characteristics of complex adaptive system, for example, the industrial cluster innovation system; therefore this paper chooses $w_{1}(t)$ and $w_{2}(t)$ as random variables to abstract such conditions. $w_{1}(t)$ and $w_{2}(t)$ have very similar statistical properties in terms of subjects' adaptability in industrial clusters, so adaptability will be regarded as the main inherent properties and introduced into the analysis of the evolutionary game process of cooperative innovation among the subjects to see whether it would have an influence on cooperative innovation strategy after the introduction. Here, based on relevant research results [3032], this paper approximates the adaptive learning process of innovation subjects in industrial cluster to Wiener process; Figure 7 is a simple form of a Wiener process.

In terms of the above system with stochastic disturbance, the existence and uniqueness of solutions meeting certain

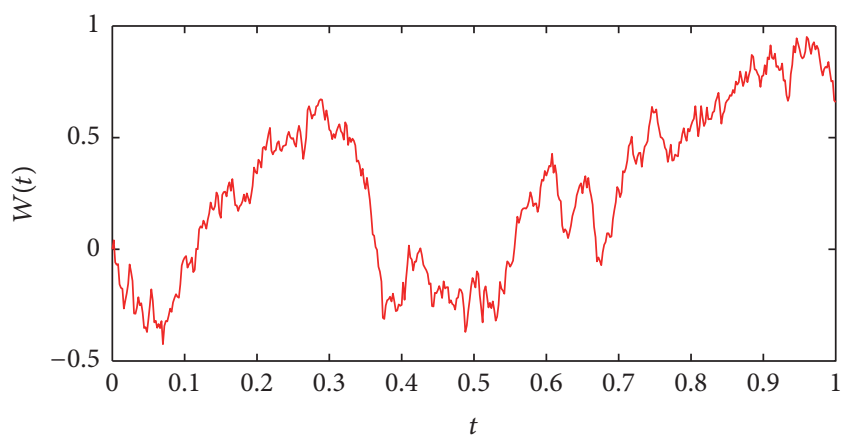

FIGURE 7: Schematic diagram of Wiener process.

conditions can be verified [33]. Due to the effect of random factors, the steady state of the stochastic equations constructed in the article cannot be discussed as a deterministic system through equilibrium, but stochastic disturbance follows certain statistical regularity. Therefore the solution to stochastic equations is in Markov process [34]. Meanwhile, in order to study the probability density function of the solution to stochastic equations, its corresponding Planck equation is analyzed [35]. According to literature [36], through analyzing the following stochastic differential equation, the analysis method goes the same as that of $d y(t)=y(1-y)(c x+d) d t+$ $y(1-y) y d w_{2}(t)$.

For $d x(t)=x(1-x)(a y+b) d t+x(1-x) y d w_{1}(t)$, the corresponding Fokker Plank equation is

$$
\begin{aligned}
& \frac{\partial p_{1}(x, t)}{\partial t}=-\frac{\partial}{\partial x}\left[f_{1}(x, y) p_{1}(x, t)\right]+\frac{1}{2} \\
& \cdot \frac{\partial^{2}}{\partial x^{2}}\left[g_{1}(x, y) \cdot g_{1}^{T}(x, y) p_{1}(x, t)\right] \\
& =[-(1-2 x)(a y+b)+y] p_{1}(x, t)-[x(1-x) \\
& \cdot(a y+b)-y(1-2 x) \frac{\partial p_{1}(x, t)}{\partial x} \\
& \left.+\frac{1}{2} x(1-x) y \frac{\partial^{2} p_{1}(x, t)}{\partial x^{2}}\right] .
\end{aligned}
$$

Among them, $f_{1}(x, y)=x(1-x)(a y+b), g_{1}(x, y)=x(1-$ $x) y$, and $p_{1}(x, t)$ stands for the probability density function of $x(t)$ at $t$ moment.

To know the smooth solution to the equation, we need to set $\partial p_{1}(x, t) / \partial x=0$. Assuming that $p_{1}(x)$ is the steady state solution, according to literature [37], we can get

$$
\begin{aligned}
& p_{1}(x)=\frac{N_{1}}{[x(1-x) y]^{2}} \\
& \cdot \exp \left\{2 \int_{x_{0}}^{x} \frac{s(1-s)(a y+b)}{[s(1-s) y]^{2}} d s\right\} \\
&=\frac{N_{1}}{[x(1-x) y]^{2}} \exp \left\{2 \frac{(a y+b)}{y^{2}} \int_{x_{0}}^{x} \frac{1}{s(1-s)} d s\right\}
\end{aligned}
$$


TABLE 4: The results of $U_{\mathrm{FP}}^{\prime}(x)$ under different $K_{1}$ conditions.

\begin{tabular}{|c|c|c|c|c|}
\hline$K_{1}$ & $U_{\mathrm{FP}}^{\prime}(x)$ & $U_{\mathrm{FP}}(x)$ & Minimal value & Maximal value \\
\hline$K_{1}<-2$ & $\begin{array}{l}U_{\mathrm{FP}}^{\prime}(x)>0 \\
x<\underline{2-K_{1}}\end{array}$ & Monotone increasing & $x=0^{+}$ & $x=1^{-}$ \\
\hline$-2<K_{1}<2$ & $\begin{array}{c}U_{\mathrm{FP}}^{\prime}(x)>0 ; \\
x>\frac{2-K_{1}}{4}, U_{\mathrm{FP}}^{\prime}(x)<0 .\end{array}$ & Decreasing after increasing & $\begin{array}{l}x=0^{+} \\
x=1^{-}\end{array}$ & $x=\frac{2-K_{1}}{4}$ \\
\hline$K_{1}>2$ & $U_{\mathrm{FP}}^{\prime}(x)<0$ & Monotone decreasing & $x=1^{-}$ & $x=0^{+}$ \\
\hline$K_{1}=2$ & - & - & $\begin{array}{l}\text { All values are } \\
\text { minimal values }\end{array}$ & - \\
\hline$K_{1}=-2$ & - & - & $\begin{array}{l}\text { Minimal values } \\
\text { cannot be achieved }\end{array}$ & - \\
\hline
\end{tabular}

Note: the range of the value of $x$ in the table is $(0,1)$.

$$
\begin{aligned}
& =\frac{N_{1}}{[x(1-x) y]^{2}} \\
& \cdot \exp \left\{2 \frac{(a y+b)}{y^{2}} \int_{x_{0}}^{x}[\ln x-\ln (1-x)+c]\right\} \\
& =\frac{N_{1}}{[x(1-x) y]^{2}}\left\{\frac{x\left(1-x_{0}\right)}{(1-x) x_{0}}\right\}^{2(a y+b) / y^{2}}
\end{aligned}
$$

We set $K_{1}=2(a y+b) / y^{2}$ and $N_{1}$ is the normalization coefficient, so the above formula can be transformed into

$$
p_{1}(x)=\frac{N_{1}}{[x(1-x) y]^{2}}\left\{\frac{x\left(1-x_{0}\right)}{(1-x) x_{0}}\right\}^{K_{1}} .
$$

Based on the literature [38], nonequilibrium phase change of the stochastic system can be achieved through the study of extreme value of random potential function. Thus, we first calculate the equation of potential function. Combining the analysis of stochastic differential equation and FokkerPlanck equation above, based on standard Wiener process, $D=0.5$ can be reached. Thus, the potential function of the original equation is

$$
\begin{aligned}
\phi(x)= & \frac{\ln p_{1}(x)}{2} \\
= & \frac{\ln N_{1}-2 \ln y+K_{1} \ln \left(1-x_{0}\right)-K_{1} \ln x_{0}}{2} \\
& +\ln x+\ln (1-x)-\frac{K_{1}}{2} \ln x \\
& +\frac{K_{1}}{2} \ln (1-x) .
\end{aligned}
$$

According to the literature [39] and the basic principle of cusp catastrophic model, through derivation, we can get the extreme value point

$$
U_{\mathrm{FP}}^{\prime}(x)=\frac{4 x+K_{1}-2}{2 x(x-1)} .
$$

According to its connotation, $x \in[0,1]$. When $x=0$ and $x=1, U_{\mathrm{FP}}^{\prime}(x)$ is singular, and the nature of $U_{\mathrm{FP}}(x)$ changes greatly, which will cause a series of phase changes. Therefore, considering the situation of $x \neq 0$ and $x \neq 1$, because of the uncertainty of the value of $K_{1}$, we get Table 4 .

The mutations of mode correspond with probability density function produced at the minimum point of potential function [40], which means that if $K_{1}<-2$, then $x=$ $0^{+}$is the attractor of system (2). And all probability will be concentrated at $x=0^{+}$, so that stochastic disturbance has no impact on the final strategy choice. The strategy of imitation, namely, which takes $x$ percent, is never selected or once selected and later abandoned. When $-2<K_{1}<$ 2 , the probability is still concentrated at $x=0^{+}$, and the probability peak began to appear at $x=1^{-}$, which means that the stochastic disturbance does influence the strategy choice and the stable state of the system polarizes (all selected or else all abandoned). When increased to $K_{1}>2$, the probability is also concentrated at $x=1^{-}$, but the probability at $x=0^{+}$gradually becomes zero; it shows that the impact of stochastic disturbance makes the strategy taking $x$ percent selected more easily.

The difference between $U(x)$ and $U_{\mathrm{FP}}(x)$ shows that multiplicative noise caused by the introduction of random driving factors will inevitably lead to different extreme value point, thus making the phase change of the deterministic system have greater changes. Using the same method of analysis, it can be found that the same is true for $d y(t)=$ $y(1-y)(c x+d) d t+y(1-y) y d w_{2}(t)$. We can then come to the conclusion that, considering the effect of stochastic disturbances in the system under certain conditions, the random system that generated the phase transition behavior becomes complicated, which means that the stable state of the strategy selected by the players generates bifurcation caused by white Gaussian noise (i.e., subjects' adaptability in industrial clusters). In addition, the steady state of the system is mainly concentrated on 0 or 1 , without considering the stochastic disturbance.

4.3. Application of Stochastic Evolutionary Game Model of Cooperative Innovation. Based on static repeated game in Section 3.1, the nonlinear characteristic is introduced to 
the evolutionary game process between subjects. Through deformation of differential equation, combined with the interaction between subjects, we add a Gaussian white noise stochastic disturbance; then the game system is transformed as follows:

$$
\begin{aligned}
d x(t)= & x(1-x)(a y+b) d t \\
& +x(1-x) y d w_{1}(t) \\
d y(t)= & y(1-y)(c x+d) d t \\
& +y(1-y) y d w_{2}(t)
\end{aligned}
$$

$d w_{1}(t) d w_{2}(t)=d t$.

What is noted is the demonstration of the economic implication of $w_{1}(t)$ and $w_{2}(t)$. Complex adaptive system of the industrial cluster determines that, in the process of cooperative innovation evolution, the subjects will affect the process of cooperative innovation. This article uses quantitative characterization to illustrate the adaptability, which is $w_{1}(t)$ and $w_{2}(t)$, the random driving forces in the evolutionary dynamics, so that their adaptability can be introduced to analyze the game process between innovation subjects. Here, the adaptive process of innovative subjects is made by approximate treatment and regarded as the wiener process.

Because the analytical solution cannot be reached, through explicitly forward Euler numerical method [41], we can simulate and analyze the effects of randomness caused by the introduction of subjects' adaptability.

For the stochastic equations, they are discussed in $T$ time units and separated in $N$ equal discrete, so the step length is $H=T / N$ and time node is $t_{n}=n H, n=0,1, \ldots, N$, which can be regarded as $x_{n}=x\left(t_{n}\right), y_{n}=y\left(t_{n}\right), \Delta w_{1 n}=$ $w_{1}\left(t_{n}\right)-w_{1}\left(t_{n}-1\right)$, and $\Delta w_{2 n}=w_{2}\left(t_{n}\right)-w_{2}\left(t_{n-1}\right)$. Therefore, the explicit forward Euler iteration formula of the above differentiation equations is as follows:

$$
\begin{aligned}
x_{n+1}= & x_{n+1}+x_{n} \cdot\left(1-x_{n}\right) \cdot\left[y_{n} \cdot(\alpha A+\beta B)-c_{1}\right] \\
& \cdot H+x_{n} \cdot\left(1-x_{n}\right) \cdot y_{n} \cdot \Delta w_{1 n} \\
y_{n+1}= & y_{n}+y_{n} \cdot\left(1-y_{n}\right) \\
& \cdot\left\{x_{n} \cdot[(1-\alpha) A+(1-\beta) B]-c_{2}\right\} \cdot H \\
& +y_{n} \cdot\left(1-y_{n}\right) \cdot x_{n} \cdot \Delta w_{2 n} .
\end{aligned}
$$

In formula (34), if items containing $\Delta w_{1 n}$ and $\Delta w_{2 n}$ are omitted, it is transformed into explicitly forward Euler iterative corresponding to the deterministic system. Coefficients of the equation here $\alpha, \beta, A, B, c_{1}$, and $c_{2}$ might as well be valued as those in Section 3.3, and the initial conditions are $x_{0}=0.6, y_{0}=0.7$. Assuming that $T=10, N=1000$, through Matlab 2014a, the Euler numerical solutions of $x$ and $y$ are simulated under the two systems, stochastic system and the corresponding deterministic system; results are shown in Figure 8.

In Figure 8, the left one is the graphic following the introduction of adaptive stochastic process, and the right one is the graphic without introducing randomness. Comparing the above two graphics, we can get the following conclusions.

Conclusion one is the final decision results after introducing the adaptive innovation subjects. After introducing the adaptability, the final steady state of $x$ and $y$ remains unchanged. Therefore, in the process of the game, adaptability can produce certain effects, but the subjects' ultimate choices of strategy will not significantly affect the results.

Conclusion two is the complexity in the cooperative innovation process. Before the equilibrium state of evolutionary game, stochastic evolutionary game curves have more twists and turns, and the traditional evolutionary game curve is more smooth, which depicts that, after the introduction of subjects' adaptability, cooperative interactions among subjects in industrial clusters exhibit nonlinear characteristics; namely, innovation strategy selection process becomes complicated, showing complex behavioral characteristics under subjects' adaptability.

Conclusion three is the time to achieve steady state changes. From the figure, although both cases reach the same final steady state, the time of their arrivals in the final steady state differs. When adaptability functions, participants in the game are able to make their final decisions in a short time; but without adaptability, subjects require more time before making their final decisions. The specific time comparison is shown in Table 5. The result shows that, in the decision process of cooperative innovation within the cluster, the innovation subjects take the subjects' adaptability into account; the stochastic evolutionary game uses shorter time to achieve at the equilibrium of the game than traditional evolutionary game, which indicates that subjects' adaptability does have an impact on cooperative innovation.

Meanwhile, the results of the study can be expanded, both in human society and in other biological environments. From a low level of microbes to a high level of mammals, cooperative behavior is a universal phenomenon, because cooperation improves the survival chances of the biological population, and also contributes to the adaptation and transformation processes of the living environment. If only in accordance with the principle of natural selection proposed by Darwin, organisms tend to choose betrayal strategy which guarantees that they can maximize self-interest. Of course, choosing to betray will bring damage to the overall interest, and the extent of such damage will deepen as the number of organisms selecting betrayal strategies increases, thereby, damaging their interest from the overall and individual level. This situation is in contrast with the reality in terms of cooperative behaviors. Therefore, we need to explore its internal mechanism. Nowak [42] and other scholars have answered this question and put forward that out of natural selection and genetic mutation mechanism. There are other mechanisms which will make organisms with competitions attach great importance to cooperation, promoting their organization through cooperation resulting in sustained biodiversity emergence. This phenomenon can be called natural cooperation. In addition, he made a thorough description mechanism promoting cooperative behaviors including kin selection, direct and indirect reciprocity, group selection, and network reciprocity. Those five mechanisms and interactive 


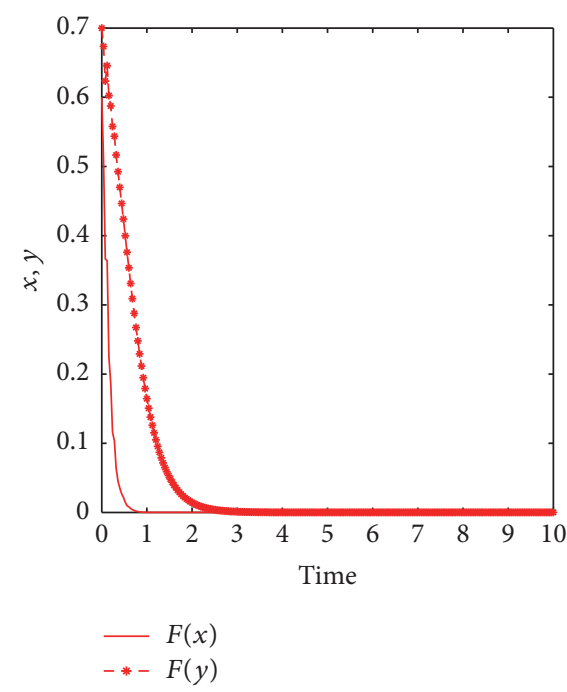

(a) Stochastic game result

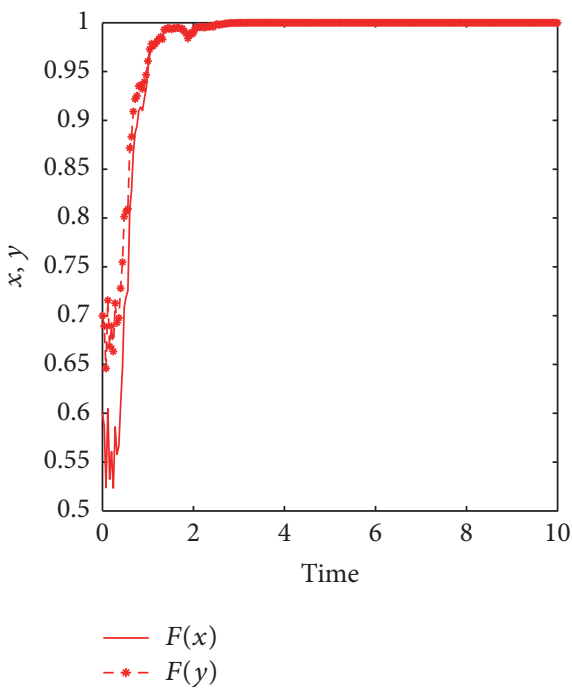

(c) Stochastic game result

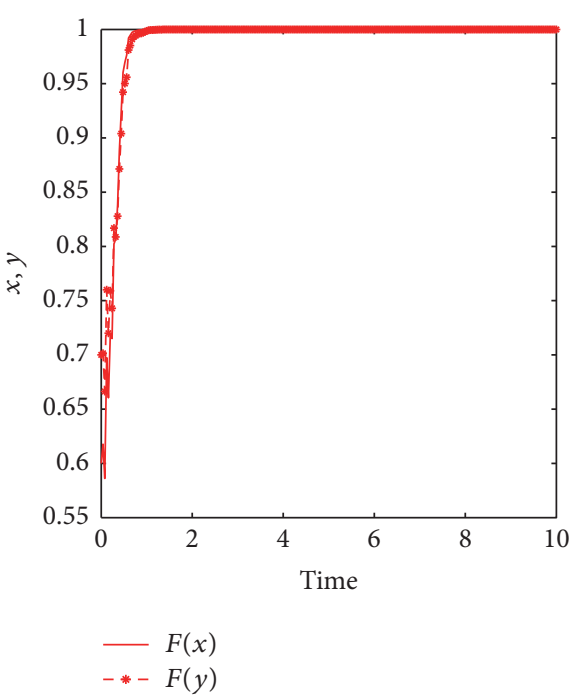

(e) Stochastic game result

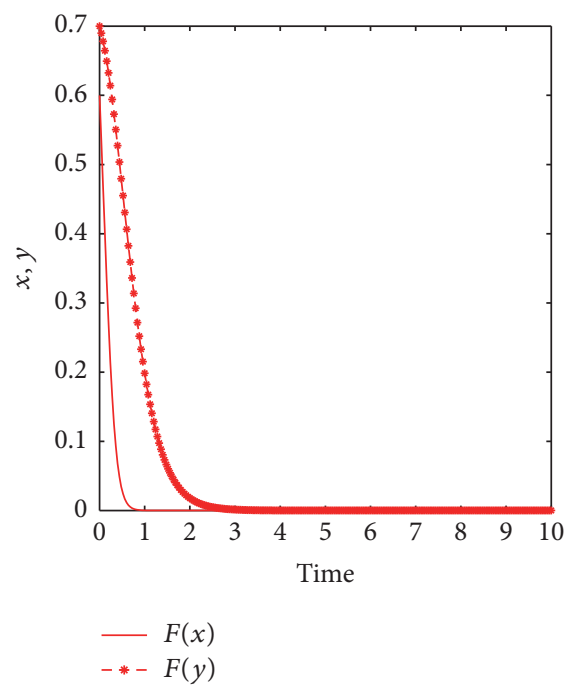

(b) Evolutionary game result

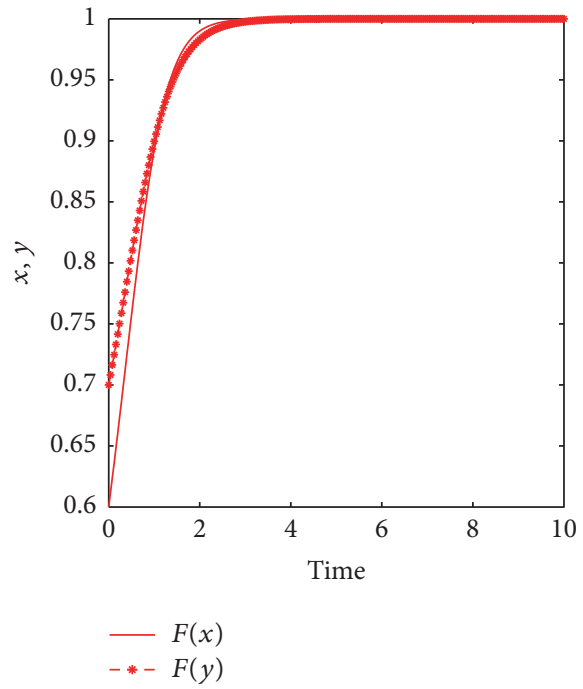

(d) Evolutionary game result

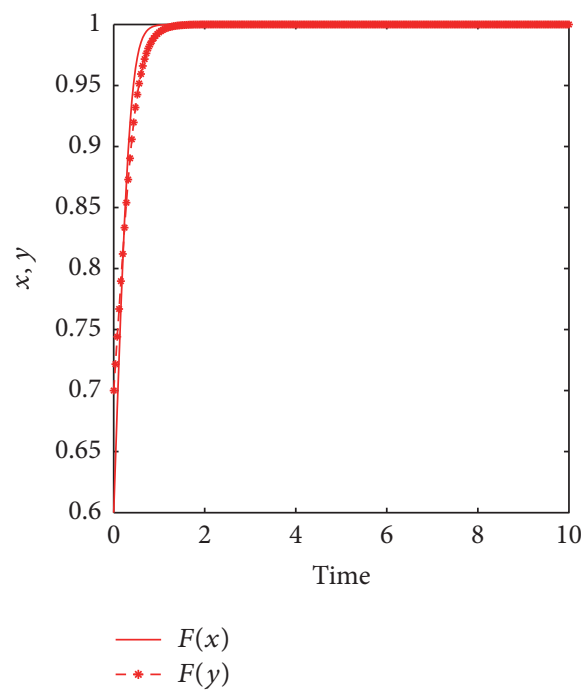

(f) Evolutionary game result

FIGURE 8: Result comparison of stochastic game result and game under definite condition. 
TABLE 5: Required time comparison of stochastic game result and game under definite condition.

\begin{tabular}{lcccccc}
\hline Strategy ratio & \multicolumn{2}{c}{ Situation 1 } & \multicolumn{2}{c}{ Situation 2 } & \multicolumn{2}{c}{ Situation 3 } \\
(a) Stochastic & (b) Game under \\
game & definite condition & (c) Stochastic & (d) Game under & (e) Stochastic & $\begin{array}{c}\text { (f) Game under } \\
\text { definite condition }\end{array}$ \\
\hline$x$ & 0.68 & 0.72 & 1.44 & 2.28 & 0.68 & 0.76 \\
$y$ & 2.40 & 2.52 & 2.12 & 2.64 & 0.80 & 1.08 \\
\hline
\end{tabular}

cooperation behaviors studied in this paper are essentially associated; for example, from the perspective of cooperative innovation, interactive cooperation behavior can be regarded as a concrete form of the reciprocity, whose repetition is the foundation of the emergence and complexity of cooperative behaviors; then what rules could the individual choose to make the two sides cooperate with each other? "Repeated Prisoner's Dilemma" computer experiments proposed by Axelrod gave answers to this issue: Setting double static "Prisoner's Dilemma" experimental conditions, by comparing different game strategies, under the "tit for tat" strategy conditions, either party in the game will not have a higher income than the other, and the maximum income limit is that both sides in the game have the same income, but the two parties under this strategy will yield the highest total revenue. Therefore, "tit for tat" rule has a good foundation for the existence, because it can guarantee that the other party has a higher yield as has been shown in Figure 9. "Tit for tat" rules in real life can be well represented and "reciprocate" and other popular proverbs contain similar principles. Although the experiments described by Axelrod demonstrated that "tit for tat" rule ensures that the bilateral cooperation has become the optimal strategy, which will be renewed, the nature of the root causes of cooperative behavior needs to be explained in a more detailed way. For example, in reality, the number of individuals at a given point in the game is uncertain, and there is no guarantee that the individual will always produce the same individual relationship in the game. In fact, the connection among individuals exhibit more network features, in which individuals' final strategy adoption involves a wider range of factors. Thus it should be considered from the point of reciprocity, using network systems to demonstrate the performance of a large number of affiliated individuals, and the network structure will become an important basis for the emergence of cooperation.

In short, the traditional evolutionary game focuses only on the final strategy choice, ignoring the stochastic disturbance caused by intrinsic properties of the parties. Stochastic evolutionary game model constructed in this paper introduces the stochastic disturbance, summarized as Gaussian white noise, into the game system as the random disturbance item. The results show that the introduction of random factors did not impact on the game strategy selection. From the process of the game, after introducing stochastic disturbance, strategy selection process fluctuates, leading to differences in terms of the rate reaching the final strategy. This result is closer to strategy selection process in realistic complex systems or complex networks and can be used as reference to the regulation of the game process.

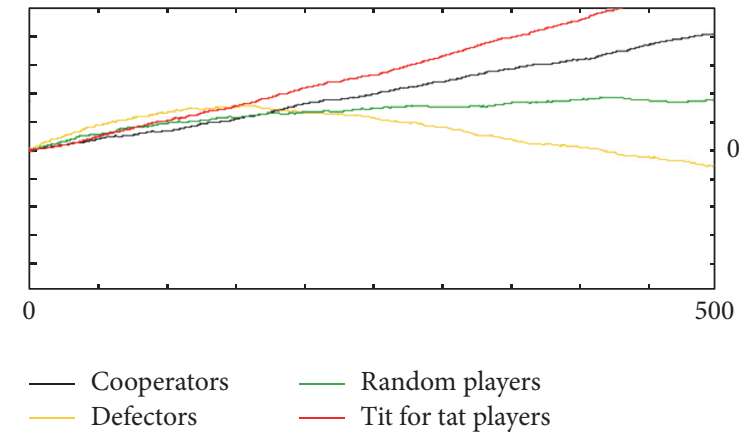

Figure 9: Result of static Prisoner's Dilemma. Source: http://systems-sciences.uni-graz.at/etextbook/gametheory/prisonersdilemma.html.

From the perspective of cooperative innovation among subjects in industrial cluster, this paper analyzes the costs and gains of cooperative innovation and the amount of government support and other external conditions remain the most important basis for cooperative innovation decisions; the subjects' inherent adaptability will have an impact on cooperative innovation strategies, but just to promote the participants to make faster decisions, and will not affect the final decision results. When it comes to the subjects in the experiment, enterprises in industrial cluster can raise their awareness of cooperative innovation and enhance learning and absorptive capacity and their other properties to reduce costs and promote earlier cooperative innovation. In short, in the cooperative innovation strategy selection process among subjects in industrial clusters, by taking their own and external factors into account, they tend to choose a more favorable strategy bringing the two sides to reach the equilibrium of the game.

\section{Case Study}

This section will select Zhongguancun as a case to study its cooperation and innovation practice. From the perspective of feasibility, this paper will only elaborate factors involved in the traditional evolutionary game model.

5.1. Cooperative Innovation Model in Zhongguancun. With reference to the research results from Wang et al. [43], Zhongguancun has formed a relatively complete multiagent cooperative innovation mode, consisting of government, universities, and research institutions, technology service agencies and science and technology enterprises, and so forth. But there are other specific innovative forms of cooperation. Through complementary exchange of technology, 
finance, policy, and other innovative resources, a relatively complete solid collaborative innovation framework has been established. Within the system, subjects gradually enhance linkages to stimulate their own advantages, pushing the overall innovation and development of Zhongguancun in different ways and at different levels. Here, this paper will analyze the relationship among different types of subjects in Zhongguancun.

(1) Cooperation among Enterprises. Partnerships between enterprises have wide channels and are in various forms, involving formal and informal forms of cooperation. When it comes to enterprises in Zhongguancun, in addition to the common formal cooperation, they often use the informal manner to achieve mutual cooperation and communication, and more and more enterprises begin face-to-face communication informally, thereby having faster and more direct access to knowledge or information. Thanks to various types of trading venues, specialized markets, seminars, and exhibitions offered by Zhongguancun, the development of such kind of informal cooperation has grown fast. The broad space provided by these platforms, entrepreneurs, technical staff, or agency personnel can be easily completed contacts and exchanges.

(2) Cooperation between Enterprises and Research Institutions. Firstly, enterprises ran by schools: the current value of Lenovo, Founder, and other leading companies, initially relying on Beijing University, Tsinghua University, and the Chinese Academy of Science has grown to billions of dollars. During the start-up period, these companies have achieved support from those institutions, as well as universities and research institution. And thus they have completed a lot of technological industrialization. Secondly, joint technological development: the natural geographical proximity between enterprises in Zhongguancun and universities and research institutions does exist. If there is a certain technical requirement in common, they will conduct technological research by means of technological innovation projects, using joint resources of both sides. Thirdly, other ways are that they mainly appear in areas of personnel exchanges in innovation and cooperation. Companies invite researchers as technical directors and personnel to undertake part-time research and select personnel to enter college for further study, and business management and technical R\&D personnel establish contacts with universities and research institutions through various formal or informal ways, which will form a sustainable, healthy exchange of information resources among enterprises, universities, and research institutions and accelerate the transformation of technological achievements by promoting the flow of knowledge.

(3) Collaboration between Enterprises and Government. Firstly, policy support: based on the enterprises' need of innovation, Zhongguancun Management Committee and other government departments lay down rules for talent introduction, tax breaks, and other aspects, which have a direct or indirect role in promoting enterprise innovation activities; for example, tax reduction and free policy offer direct assistance to SMEs during the establishment and start-up of their development. Secondly, financial support: particularly at the initial stage of cooperative innovation, as financial institutions lack willingness to support them, direct financial support or financing channels from government will have a very significant positive effect. Thirdly, other forms: a wide range of government-led products launches; product Expo will help enterprises in Zhongguancun to strengthen exchanges with outside markets, accelerating the transmission and agglomeration of knowledge and information.

(4) Cooperation between Enterprises and Intermediaries. Such kind of cooperation helps companies to be informed of market information and policy changes. From the current development of Zhongguancun, the form of cooperation between enterprises and intermediaries lies in enterprises entering into the industrial associations and other institutions, set up after the seminar through intermediaries, and then a platform is established to promote the exchange of information between members through seminars, reports, and conferences. In recent years, Zhongguancun has strengthened the construction of technical service mediation, allowing intermediary agencies to offer more services in terms of technical innovation and enterprises' business management.

5.2. Analysis on Game Factors of Cooperative Innovation in Zhongguancun. Combining the cooperative innovation evolutionary game model in industrial cluster constructed above, an analysis will be made from the factors of evolutionary game in Zhongguancun's cooperative innovation practice.

(1) The Cost of Cooperative Innovation $c$. From the foregoing model simulation results, reducing the cost of cooperative innovation $c$ can promote subjects to select cooperative innovation strategy. When it comes to Zhongguancun, cooperative innovation practice is mainly in the following aspects. Firstly, Zhongguancun outstanding industrial agglomeration advantages reduce the cost of innovation. In its development and growth process, science and technology enterprises continued to gather in Zhongguancun: based on the "Electronics Street," it continued to improve their innovative ability and is now becoming a national independent innovation demonstration zone, through continuous optimization of its own industrial space layout, for example, from "one area and ten parks" to "one area and sixteen parks," gathering more technological innovation industries; currently Zhongguancun already has six leading industries and four potential industries, and the industrial chain has helped to carry out innovation activities: with the innovative enterprises as the pioneers, it inspires upstream and downstream enterprises to drive on interactive cooperation, while achieving horizontal cooperation using innovative platform. In addition, SMEs cater to the increasing need of market innovation through the establishment of cooperative alliances. Secondly, Zhongguancun science and technology innovation and financial support can reduce financing costs. When technology and science finance provides various forms of financial support to enterprises, especially SMEs, these enterprises will not only benefit from investment but reflect their latest service 
demand, thus promoting innovation in technology and financial services and financial instruments. Currently, Zhongguancun science and technology financial system includes a base (enterprise credit system construction), six mechanisms (six mechanisms for technology and capital efficient connection), and ten channels (angel investors, secured financing, public financing of investment, and other ways), to ensure adequate support from the government and social capital, while continuing to develop a variety of new means of equity financing and debt financing to meet the needs of SMEs. Through financing, new requirements for corporate financial service will continue to be collected to improve relevant financial platform tools, such as share transfer agent system and other means of financing, which will support the practice of promoting the rapid upgrading of Zhongguancun science financial services. Thirdly, specialized and innovative services provided by science and technology intermediary organizations can reduce the cost of innovation risk. The intermediary organizations mainly provide full cycle and full range of innovative services for Zhongguancun hightech SMEs: relying on its own professionals, legal knowledge, and other advantageous resources to provide information consultation for enterprises; based on technological transaction, quality certification, and so forth to offer enterprises technology related services; providing financial services to enterprises through credit rating, financing guarantees, and other channels; relying on industrial associations and other industrial alliances to promote the industry to upgrade its management level and strengthen its awareness of selfdiscipline.

(2) Cooperative Innovation Gains B. From the foregoing model simulation results, increased cooperative innovation gains $B$ can promote subjects to select cooperative innovation strategy. When it comes to Zhongguancun, cooperative innovation is mainly reflected in the establishment of alliances among industries, universities, and research institutions, which improves the innovation efficiency and increases the innovation gains. Zhongguancun has obvious geographical advantages; it is within the most intensive area of universities and research institutions in Beijing, which provides unique natural conditions for various forms of cooperation and innovation research; besides, the number of national laboratories, engineering research centers, and science and technology park of universities is gradually increasing in Zhongguancun, and the establishment of cooperative innovation alliance can provide the most basic conditions for the wealthiest technical innovations; after years of practice, Zhongguancun has produced a wide range of innovative forms of cooperation, such as building joint laboratories and joint research projects, and continues to explore the cultivation and communication mode of scientific and technological talents, among which laboratories, incubators, and science parks are the most representative initiatives. Figure 10 shows several typical cooperative innovation modes and corresponding examples in Zhongguancun.

(3) The Amount of Government Support S. From the foregoing model simulation results, increased amount of government support $S$ can promote subjects to select cooperative innovation strategy. Deep into cooperative innovation practice Zhongguancun, government support for cooperative innovation is mainly reflected in that enterprises can take advantage of the Zhongguancun Science and Technology Innovation and Industry Promotion Center (Promotion Center) and propose suggestions for the improvement of innovative support policies, which prompts the government to constantly improve their ways of cooperation and innovation support: firstly, by means of government procurement to expand market in terms of the scope of products and services of respective enterprises; secondly, through the promotion of the center described previously to actively encourage financial institutions, industry, universities, and research organizations and other types of technology intermediary body to be integrated into Zhongguancun overall cooperative innovation system. With "policy making $\rightarrow$ policy feedback $\rightarrow$ policy improvement" interactive support process, government can precisely guide and encourage cooperative innovation. From the current practice in Zhongguancun, government guidance has gained significant results.

Through the above analysis, the reason why cooperative innovation in Zhongguancun is able to achieve healthy and sustainable development lies in that the cooperative innovation model implemented is in line with the requirements of the law of development economics; namely, from evolutionary game model, these models can induce the body to choose cooperative innovation strategy, thus promoting subjects not only to conduct innovation activities independently but also to work jointly. This course of action laid the foundation for the formation of innovation networks and provided the conditions for more innovation outputs. In addition, by stochastic evolutionary game model, we know that the introduction of subjects' adaptability will not affect the final choice of innovative strategies industrial clusters but can reduce the time of selection. Therefore, in the future cooperative innovation in Zhongguancun, as the number and types of subjects involved in innovation, more attention should be paid to the heterogeneity of the subjects, focusing on stimulating the subjects' own will and power of innovation and creating a good innovation support environment, so as to continuously enhance their adaptability to the environment and promote more efficient forms of cooperative innovation.

\section{Conclusion}

Firstly, adaptive learning mechanism of the subject is the research foundation of industrial cluster innovation. With the accumulated innovation activities created by the subjects, the new trend of innovation would be formed inside the cluster and improve the innovative capacity of the cluster at the same time. On the other side, the innovative capacity of the cluster determines the individual innovative subjects' behavior. If the industrial cluster subjects accumulate innovative knowledge via efficient and continuous learning activities, the total amount of innovative knowledge of the industrial cluster would increase automatically and correspondingly. As a result, the entire cluster members would benefit from the accumulated innovative knowledge and pushed to create 


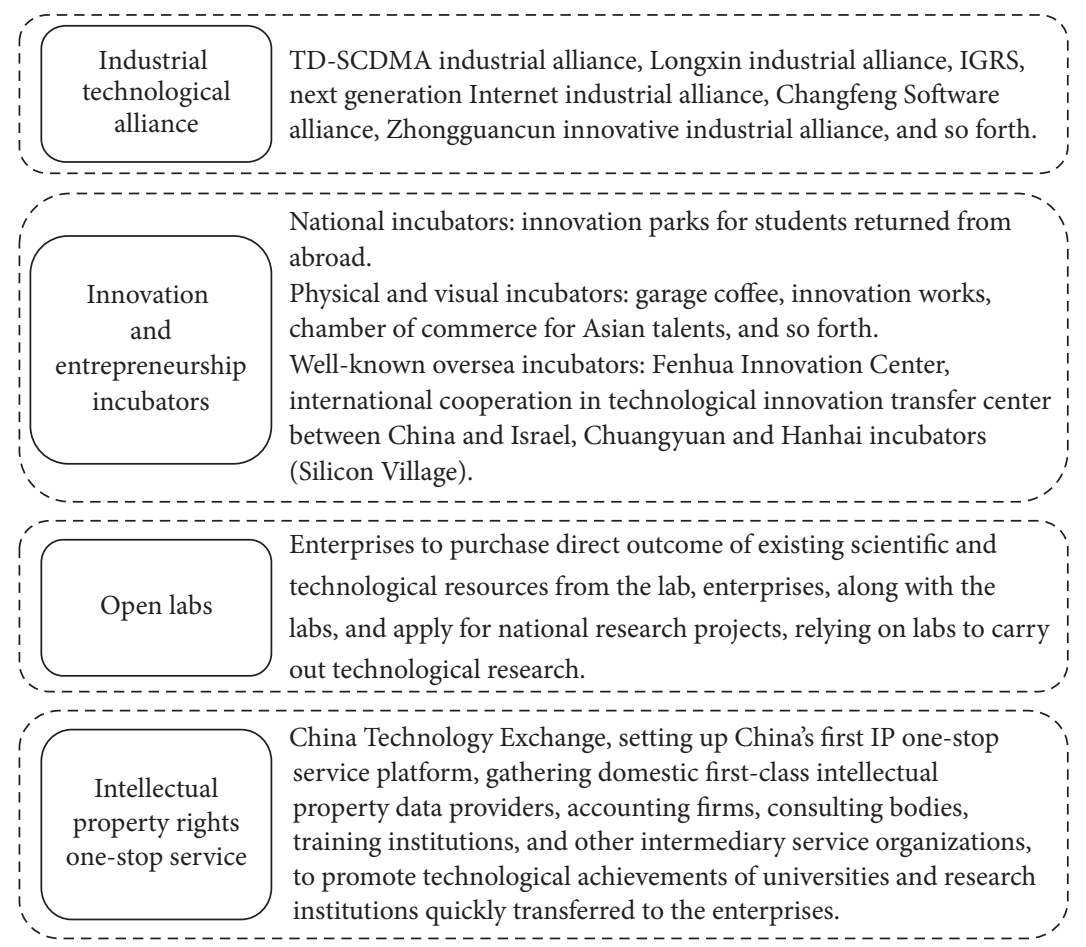

FIgURE 10: Typical modes of cooperation innovation of Zhongguancun.

more value during the entire manufacture procedure [44]. Considering the various changes from the individual or cluster's perspective, it would bring out various possibilities, which would accidentally promote the development route of either enterprise or the entire industrial cluster. Even an occasional hint may influence the subject's direction and the distribution of the organism in the coming future, especially the situations with high internal resonance [45]. There is a hidden fact that each subject would show individual innovative capacity and lead to new innovation behavior, which would create value from innovation. As the information of successful innovation value creation spreading to other subjects, the learning and imitation effects would come out inside the cluster. This phenomenon may lead to a different development direction and moving route intentionally or unintentionally. But if other subjects have made huge changes relevantly, the change of industrial cluster's development direction would be pushed, and new advantages of the cluster will be formed as well.

Secondly, bounded rationality determines that subjects cannot find the optimal strategy in line with their own interests in the initial stage of the game but can adjust to identify a relatively good strategy through repeated trial and error learning; bounded rationality means that at least some of the corporates cannot select completely rational equilibrium strategy, and the final equilibrium is obtained through continuous adjustment, which cannot be determined once, even if the game has reached equilibrium, but there are still possible deviations; if there is a real equilibrium in the framework of bounded rationality, it is achieved by the two sides after continuous learning and adjustment, which can withstand minor disturbance and can be adjusted again and "restored" to the balanced and stable state.

In short, the traditional evolutionary game focuses only on the final strategy choice, without taking into account stochastic disturbance, the intrinsic properties of the subjects in the game. Stochastic evolutionary game model constructed in this paper summarizes the random factors as Gaussian white noise and brings it into the game system as the stochastic disturbance item. The results show that the introduction of random factors did not impact on the game strategy selection results of the two sides; from the process of the game, with stochastic disturbance factors, strategy selection process fluctuates, leading to differences in the rate to reach the final strategy, which is closer to the strategy selection process in complex systems or complex networks, and can be used as reference for the process regulation. In future studies, we will further combine the network structure in industrial clusters with adaptive and game behaviors of subjects to construct multiagent innovation system in industrial clusters based on complex adaptability, using systems emergence and innovation network theory at a more macrolevel.

From the perspective of cooperative innovation behaviors of subjects in industrial cluster, the costs and gains of cooperative innovation and external conditions such as the amount of government support are still the most important basis for decision-making in cooperative innovation in industrial clusters; inherent adaptability would impact on cooperative innovation strategy, but merely to promote them to make faster decisions in the game, and will not affect the final decision results. When it comes to the subjects in the experiment, enterprises in industrial cluster can raise their 
awareness of cooperative innovation and enhance learning and absorptive capacity and their other properties to reduce costs and promote earlier cooperative innovation. In short, in the cooperative innovation strategy selection process among subjects in industrial clusters, by taking their own and external factors into account, they tend to choose a more favorable strategy bringing the two sides to reach the equilibrium of the game.

\section{Competing Interests}

The authors declare that regarding the publication of this article there are no competing interests.

\section{References}

[1] R. Martin and P. Sunley, "Conceptualizing cluster evolution: beyond the life cycle model?” Regional Studies, vol. 45, no. 10, pp. 1299-1318, 2011.

[2] J. H. Holland, Hidden Order: How Adaptation Builds Complexity, Addison-Wesley, Boston, Mass, USA, 1995.

[3] H. A. Simon, Administrative Behavior-A Study of DecisionMaking Processes in Administrative Organization, Free Press, New York, NY, USA, 1976.

[4] T. Brenner and S. Greif, "The dependence of innovativeness on the local firm population: an empirical study of German patents," Industry and Innovation, vol. 13, no. 1, pp. 21-39, 2006.

[5] R. Garcia, "Uses of agent-based modeling in innovation/new product development research," Journal of Product Innovation Management, vol. 22, no. 5, pp. 380-398, 2005.

[6] E. H. Hu, Theoretical and Empirical Research on Innovation Behavior of Enterprise Clusters-Based on Complex Adaptive System Theory, Science Press, Beijing, China, 2007.

[7] C. Suematsu, Transaction Cost Management, Springer International, Cham, Switzerland, 2014.

[8] T. Broekel, "Do cooperative research and development (R\&D) subsidies stimulate regional innovation efficiency? Evidence from Germany," Regional Studies, vol. 49, no. 7, pp. 1087-1110, 2015.

[9] M. Expósito-langa, J.-V. Tomás-Miquel, and F. X. MolinaMorales, "Innovation in clusters: exploration capacity, networking intensity and external resources," Journal of Organizational Change Management, vol. 28, no. 1, pp. 26-42, 2015.

[10] X. Zhang, Z. Zheng, K. Huang, and P. Wang, “Organizational culture, inter-organizational learning ability and innovation performance of the technology alliance of small and medium enterprises," in Proceedings of the IEEE International Conference on Industrial Engineering and Engineering Management, vol. 87, pp. 29-33, Bangkok, Thailand, December 2013.

[11] E. L. Paiva, L. C. D’Avila, and I. Gavronski, “The relationship between manufacturing integration and performance from an activity-oriented perspective," BAR-Brazilian Administration Review, vol. 8, no. 4, pp. 376-394, 2011.

[12] E. Fakhrutdinova, S. Mokichev, and J. Kolesnikova, "The influence of cooperative connections on innovation activities of enterprises," World Applied Sciences Journal, vol. 27, no. 2, pp. 212-215, 2013.

[13] H. Ya-Pin and H. Long-Ying, "Research on asymmetric trust evolution in the complex products and systems cooperative innovation network," in Proceedings of the 18th Annual International Conference on Management Science and Engineering (ICMSE '11), pp. 313-320, IEEE, Rome, Italy, September 2011.

[14] E. Alvarez-Garrido and G. Dushnitsky, "Are entrepreneurial venture's innovation rates sensitive to investor complementary assets? Comparing biotech ventures backed by corporate and independent VCs," Strategic Management Journal, vol. 37, no. 5, pp. 819-834, 2016.

[15] R. Motzek, Motivation in Open Innovation, AV Akademikerverlag GmbH \& Co. KG, Saarbrücken, Germany, 2012.

[16] R. Sethi and E. Somanathan, "Understanding reciprocity," Journal of Economic Behavior \& Organization, vol. 50, no. 1, pp. $1-27,2003$.

[17] V. Anderhub, D. Engelmann, and W. Güth, "An experimental study of the repeated trust game with incomplete information," Journal of Economic Behavior \& Organization, vol. 48, no. 3, pp. 197-216, 2002.

[18] M. M. Leng, Competition and Cooperation in Supply Chains: Game-Theoretic Models, McMaster University, Hamilton, Canada, 2005.

[19] A. Kydd, "Trust building, trust breaking: the dilemma of NATO enlargement," International Organization, vol. 55, no. 4, pp. 801828, 2001.

[20] J. M. Smith and G. R. Price, "The logic of animal conflict," Nature, vol. 246, no. 5427, pp. 15-18, 1973.

[21] J. W. Weibull, Evolutionary Game Theory, MIT Press, Cambridge, Mass, USA, 1995.

[22] P. D. Taylor and L. B. Jonker, "Evolutionary stable strategies and game dynamics," Mathematical Biosciences, vol. 40, no. 1-2, pp. 145-156, 1978.

[23] S. H. Jia and J. P. Yang, "The review of leading firms' stimulative effect in the evolution of industrial cluster," Review of Industrial Economics, vol. 6, no. 1, pp. 129-136, 2007.

[24] D. Friedman, "Evolutionary games in economics," Econometrica, vol. 59, no. 3, pp. 637-666, 1991.

[25] W. J. Ewens, Mathematical Population Genetics. I. Theoretical Introduction, Springer, Berlin, Germany, 2004.

[26] P. Ao, "Letter to the editor: potential in stochastic differential equations: novel construction," Journal of Physics A: Mathematical and General, vol. 37, no. 3, pp. L25-L30, 2004.

[27] P. Ao, "Laws in Darwinian evolutionary theory," Physics of Life Reviews, vol. 2, no. 2, pp. 117-156, 2005.

[28] X. Song, Landscape Construction in the Evolutionary Model, Shanghai Jiao Tong University, Shanghai, China, 2013.

[29] C. F. Ding, The Dynamical Analysis of Evolutionary Algorithm, Shanghai Jiao Tong University, Shanghai, China, 2012.

[30] V. Limic and R. Pemantle, "More rigorous results on the Kauffman-Levin model of evolution," The Annals of Probability, vol. 32, no. 3, pp. 2149-2178, 2004.

[31] J. Y. Weng, "Competition, uncertainty and inter-firm technological innovation cooperation," Economic Research Journal, vol. 3, pp. 53-60, 2002.

[32] Q. Tian, H.-Y. Liang, and M.-J. Zhou, "Research on the transaction memory system in the team of early supplier involvement," in Proceedings of the IEEE 18th International Conference on Industrial Engineering and Engineering Management (IE\&EM '11), pp. 1503-1507, IEEE, Beijing, China, September 2011.

[33] S. G. Hu, C. M. Huang, and F. K. Wu, Stochastic Differential Equation, Science Press, Beijing, China, 2008. 
[34] Z. Fan, M. Liu, and W. Cao, "Existence and uniqueness of the solutions and convergence of semi-implicit Euler methods for stochastic pantograph equations," Journal of Mathematical Analysis and Applications, vol. 325, no. 2, pp. 1142-1159, 2007.

[35] S. M. J. Lyons, S. Sarkka, and A. J. Storkey, "Series expansion approximations of brownian motion for non-linear Kalman filtering of diffusion processes," IEEE Transactions on Signal Processing, vol. 62, no. 6, pp. 1514-1524, 2014.

[36] T. C. Gard, Introduction to Stochastic Differential Equations, vol. 114 of Monographs and Textbooks in Pure and Applied Mathematics, Marcel Dekker, New York, NY, USA, 1988.

[37] N. H. Du and V. H. Sam, "Dynamics of a stochastic LotkaVolterra model perturbed by white noise," Journal of Mathematical Analysis \& Applications, vol. 324, no. 1, pp. 82-97, 2006.

[38] G. Hu, Stochastic Force and Nonlinear System, Shanghai Science and Technology Education Publishing, Shanghai, China, 1995.

[39] F. H. Lin, Catastrophe Theory and Its Application, Shanghai Jiao Tong University Press, Shanghai, China, 1987.

[40] Y. Xu, X. Zhao, and Y. Yang, "Application and research of random mutation theory," Statistics \& Decision, vol. 22, pp. 3438, 2012.

[41] C. Shi, Y. Xiao, and C. Zhang, "The convergence and MS stability of exponential euler method for semilinear stochastic differential equations," Abstract and Applied Analysis, vol. 2012, Article ID 350407, 19 pages, 2012.

[42] M. A. Nowak, "Five rules for the evolution of cooperation," Science, vol. 314, no. 5805, pp. 1560-1563, 2006.

[43] Z. T. Wang, C. S. Zhang, and Z. W. Zhang, "Characteristics and optimization of collaborative innovation model for small and medium-sized technology-based enterprise - a case study of zhongguancun," Academics, vol. 8, pp. 239-244, 2015.

[44] J. Wei, Industrial Cluster: Innovation Systems and Technological Learning, Science Press, Beijing, China, 2003.

[45] N. Wiener, Cybernetics: Or, Control and Communication in the Animal and the Machine, M.I.T. Press, Cambridge, Mass, USA, 1965. 


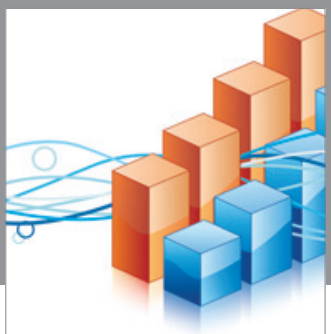

Advances in

Operations Research

vatem alat4

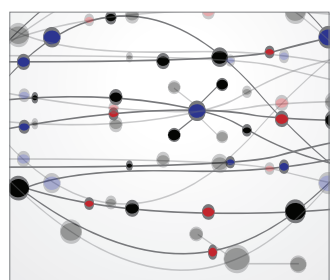

\section{The Scientific} World Journal
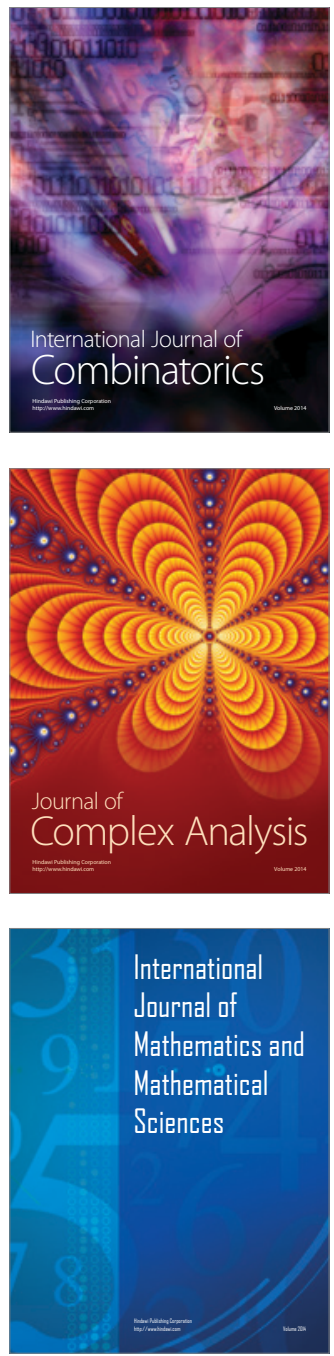
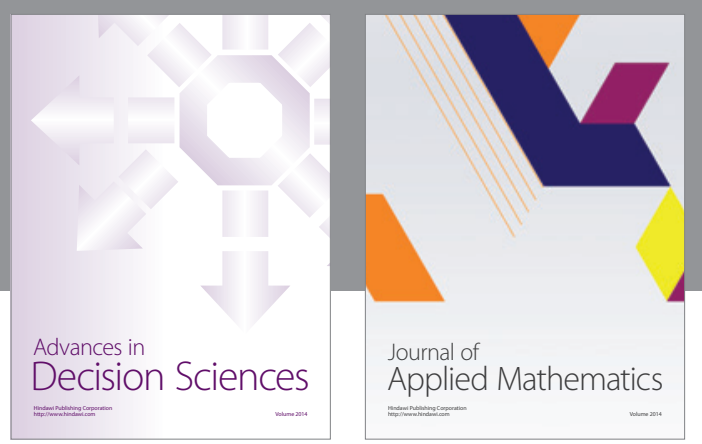

Algebra

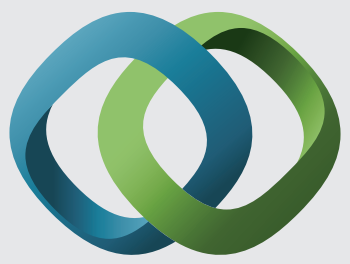

\section{Hindawi}

Submit your manuscripts at

http://www.hindawi.com
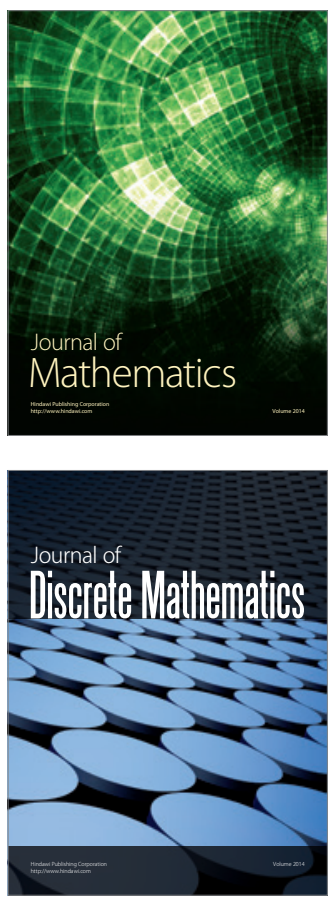

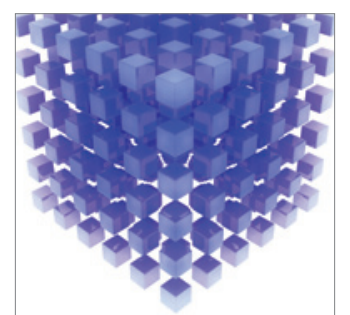

Mathematical Problems in Engineering
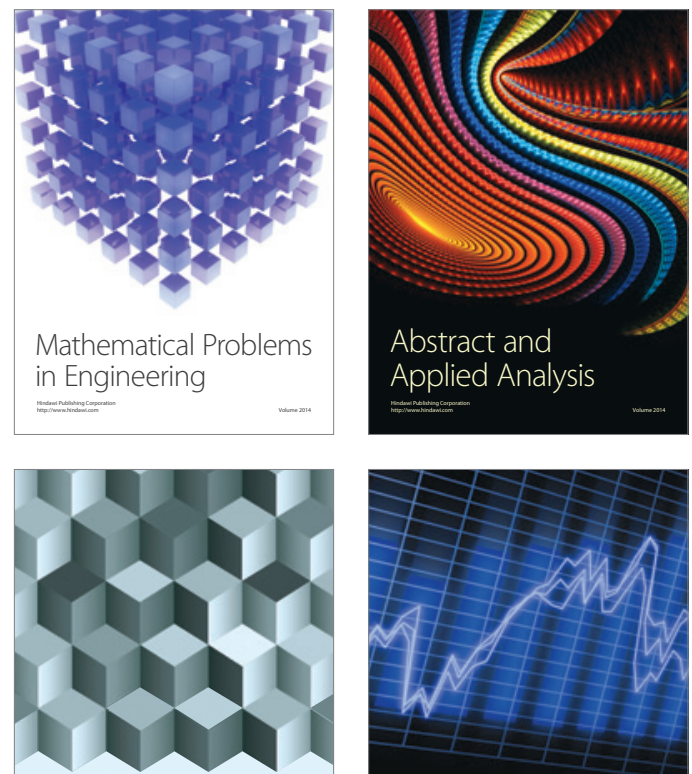

Journal of

Function Spaces

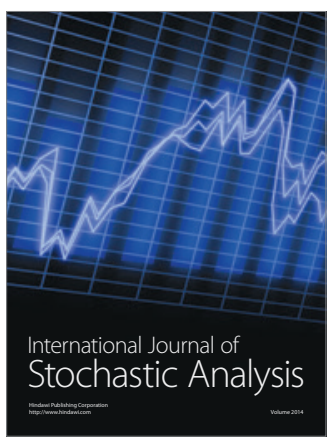

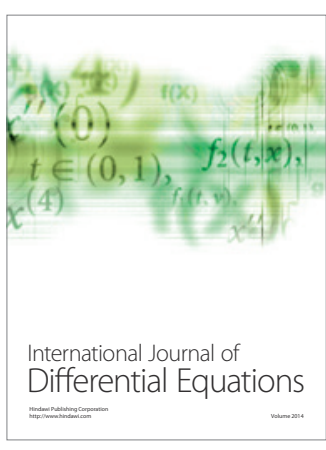
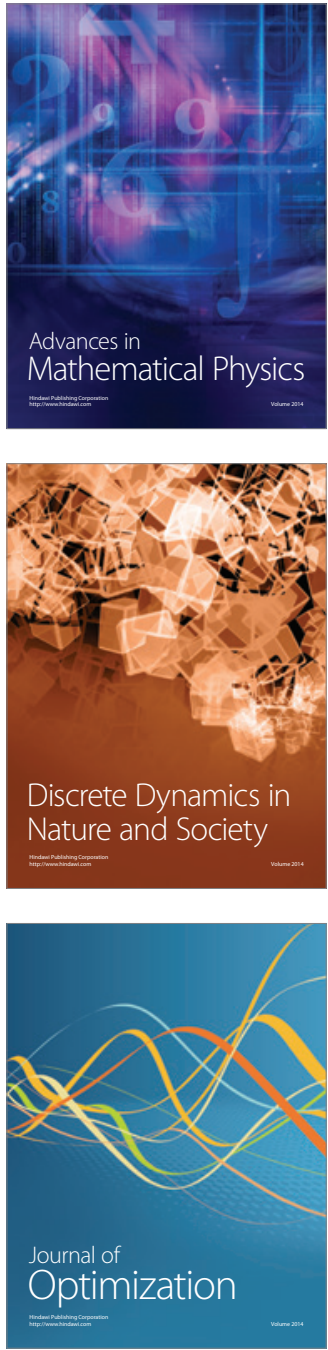